\title{
ECS-based investigation of chloroplast ATP synthase regulation
}

\author{
Felix Buchert ${ }^{1,2}$, Benjamin Bailleul ${ }^{1}$ and Pierre Joliot ${ }^{1}$
}

\author{
${ }^{1}$ Laboratoire de Physiologie Membranaire et Moléculaire du Chloroplaste-UMR7141, IBPC, CNRS- \\ Sorbonne Université, Paris, France. \\ ${ }^{2}$ Institute of Plant Biology and Biotechnology, University of Münster, Schlossplatz 8, 48143 Münster, \\ Germany.
}

\section{Correspondence :}

Felix Buchert, Institute of Plant Biology and Biotechnology, University of Münster, Schlossplatz 8, 48143 Münster, Germany,

Phone number : +49-(0)251-8324705

e-mail : f.buchert@uni-muenster.de

\section{Abstract}

The chloroplast ATP synthase $\left(\mathrm{CF}_{1} \mathrm{~F}_{0}\right)$ contains a specific feature to the green lineage: a $\gamma$-subunit redox domain which contains a cysteine couple and interacts with the torque-generating $\beta D E L S E E D-l o o p$. Based on the recently solved structure of this domain, it was proposed to function as a chock. In vitro, $\gamma$-disulfide formation slows down the activity of the $\mathrm{CF}_{1} \mathrm{~F}_{\mathrm{o}}$ at low transmembrane electrochemical proton gradient $\left(\Delta \tilde{\mu}_{\mathrm{H}^{+}}\right)$. Here, we utilize in vivo absorption spectroscopy measurements for functional $\mathrm{CF}_{1} \mathrm{~F}_{\mathrm{o}}$ activity characterization in Arabidopsis leaves. The spectroscopic method allows us to measure the $\Delta \tilde{\mu}_{\mathrm{H}^{+}}$present in dark-adapted leaves, and to identify its mitochondrial sources. Furthermore, we follow the fate of the extra $\Delta \tilde{\mu}_{\mathrm{H}^{+}}$generated by an illumination, including its osmotic and electric components, and from there we estimate the lifetime of the light-generated ATP. In contrast with a previous report [Joliot and Joliot, Biochim. Biophys. Acta, 1777 (2008) 676-683], the CF $F_{\circ} \gamma$-subunit exists mostly in an oxidized form in the dark-adapted state. To study the redox regulation of the $\mathrm{CF}_{1} \mathrm{~F}_{\mathrm{o}}$, we used thiol agent infiltration in WT and a mutant that does not form the $\gamma$-disulfide. The obtained $\Delta \tilde{\mu}_{\mathrm{H}^{+}}$-dependent $\mathrm{CF}_{1} \mathrm{~F}_{\mathrm{o}}$ activity profile in the two $\gamma$-redox states in vivo reconciles with previous biochemical in vitro findings [Junesch and Gräber, Biochim. Biophys. Acta, 893 (1987) 275-288]. The highest rates of ATP synthesis we measured in the two $\gamma$-redox state were similar at high $\Delta \tilde{\mu}_{\mathrm{H}^{+}}$. In the presence of the $\gamma$-dithiol, similar rates were obtained at a $\sim 45 \mathrm{mV}$ lower $\Delta \tilde{\mu}_{\mathrm{H}^{+}}$value compared to the oxidized state, which closely resembled the energetic gap of $0.7 \Delta \mathrm{pH}$ units reported in vitro. 
Introduction

During photosynthesis, the electron transfer is coupled to a movement of protons across the thylakoid membrane, generating an electrochemical proton gradient $\left(\Delta \tilde{\mu}_{\mathrm{H}^{+}}\right)$which consists of an osmotic component (the concentration gradient of hydrogen ions, $\Delta \mathrm{pH}$ ) and a membrane potential $(\Delta \Psi)$. The chloroplast ATP synthase $\left(\mathrm{CF}_{1} \mathrm{~F}_{\mathrm{o}}\right)$ catalyzes the conversion of the $\Delta \tilde{\mu}_{\mathrm{H}^{+}}$into the synthesis of ATP, subsequently used in the Calvin-Benson-Bassham cycle. The enzyme [recently reviewed in 1,2] consists of two portions: a membrane-spanning $F_{0}$ (subunits abb' $c_{14}$ ) and a membrane-attached $F_{1}$ (subunits $\left.\alpha_{3} \beta_{3} \gamma \delta \varepsilon\right)$. In the chloroplast enzyme of vascular plants, during a $360^{\circ}$ rotation of subunits $\gamma \varepsilon c_{14}$ against the static subunits $\alpha_{3} \beta_{3} \delta a b b^{\prime}, 14 \mathrm{H}^{+}$are translocated along the electrochemical gradient while 3 molecules of ATP are synthesized. Flexibility in the peripheral stalk might redistribute torsional energy differences during the rotation cycle [3]. Perfect coupling is observed in isolated chloroplasts, i.e., $\mathrm{H}^{+}$ slip processes do practically not occur in vitro [4, 5, for a different view see Ref. 6]. Being reversible, $\mathrm{CF}_{1} \mathrm{~F}_{\mathrm{o}}$ catalyzes both the synthesis and hydrolysis of ATP and the reaction direction is determined by the extent of the $\Delta \tilde{\mu}_{\mathrm{H}^{+}}$and the $[\mathrm{ATP}] /([\mathrm{ADP}][\mathrm{P}])$ ratio.

The activity of the $\mathrm{CF}_{1} \mathrm{~F}_{\mathrm{o}}$ plays a central role in photosynthesis; it fuels the chemical phase of photosynthesis with ATP but can also participate in the regulation of the photochemical phase by modulating the osmotic component of the $\Delta \tilde{\mu}_{\mathrm{H}^{+}}$. Indeed, the lumenal $\mathrm{pH}$ is involved in the regulation of the photosynthetic electron transfer at two levels. It regulates the photoprotective mechanism in photosystem II (PSII) and the turnover of the cytochrome $b_{6} f$ through the so-called photosynthetic control. Being at the crossroads of the dark and light phases of photosynthesis, the activity of $\mathrm{CF}_{1} \mathrm{~F}_{\mathrm{o}}$ needs to be fine-tuned and, although other regulations exist [7-9], two main regulatory mechanisms can modulate the rates of the $\mathrm{CF}_{1} \mathrm{~F}_{0}$. The first one is relevant for F-ATP synthase in general which is a regulation by its substrate, the $\Delta \tilde{\mu}_{\mathrm{H}^{+}}$. Above a light-induced threshold $\Delta \tilde{\mu}_{\mathrm{H}^{+}}$, the transition of the inactive $\mathrm{CF}_{1} \mathrm{~F}_{\mathrm{o}}$ to a fully active form was observed in isolated chloroplasts $[10,11]$. Several studies on chloroplasts and leaves demonstrated that each of the two components of the $\Delta \tilde{\mu}_{\mathrm{H}^{+}}$can activate the enzyme, provided that they allow the $\Delta \tilde{\mu}_{\mathrm{H}^{+}}$to reach this critical level [12-16]. We call this level $\Delta \tilde{\mu}_{\mathrm{H}^{+}}$activation hereafter. Specific to $\mathrm{CF}_{1} \mathrm{~F}_{\mathrm{o}}$ in the green lineage is a second, "thiol modulation" or "redox regulation" mechanism which involves an insertion in the $\gamma$-subunit. Compared to cyanobacterial ancestors, the additional domain harbors a redox-active Cys couple which forms a disulfide in the dark. Formation of the $\gamma$-disulfide is, in part, mediated by a thioredoxin-like2/2-Cys peroxiredoxin redox cascade in Arabidopsis [17]. Dithiol formation in vivo is catalyzed by thioredoxin at moderate light intensities [18] and by NADPH thioredoxin reductase $C$ in dim light [19, 20]. It was shown that the redox-active Cys is exposed to the solvent in the presence of a $\Delta \tilde{\mu}_{\mathrm{H}^{+}}[21,22]$, which could contribute to efficient dithiol formation in the presence of light $[23,24]$. Moreover, various sites in $\mathrm{CF}_{1} \mathrm{~F}_{\mathrm{o}}$, some of them located in the $\gamma$-redox domain, were chemically labeled in the presence of $\Delta \tilde{\mu}_{\mathrm{H}^{+}}[21,22,25-28$, reviewed in 29]. From a structural point of view, in the disulfide form, the $\gamma$-redox domain was found to act as an ATP hydrolysis chock that is vicinal to the torque-generating $\beta D E L S E E D-l o o p ~[3,30]$. A particular $\gamma$-hairpin structure protrudes into the $\alpha_{3} \beta_{3}$ hexamer and may interfere with efficient rotational catalysis. In the dark, when the $\Delta \tilde{\mu}_{\mathrm{H}^{+}}$is low and the disulfide is present, the slowdown of ATP hydrolysis is believed to preserve high ATP levels [31]. However, point mutants of the redox-active Cys in Arabidopsis thaliana are viable [32]. Another mutant, termed gamera, remains in the $\gamma$-dithiol state in the dark by expressing a redox-insensitive $\gamma$-subunit isoform [33] and displays a stay green phenotype after several days in the dark [34]. It was suggested that altered $\mathrm{pH}$-dependent protein import might be, in part, responsible for the phenotype. Several $\gamma$-/ $\beta$-subunit interactions, possibly the 
$\gamma$-hairpin structure as well, seem to be modulated by the $\gamma$-redox state [35]. Taken together, this suggests that $\mathrm{CF}_{1} \mathrm{~F}_{\mathrm{o}}$ experiences intertwined $\Delta \tilde{\mu}_{\mathrm{H}^{+-}}$and redox-dependent structural changes. Junesch and Gräber demonstrated in vitro that the dependency between the redox and $\Delta \tilde{\mu}_{\mathrm{H}^{+}}$regulations of $\mathrm{CF}_{1} \mathrm{~F}_{\mathrm{o}}$ stems from a lower $\Delta \tilde{\mu}_{\mathrm{H}^{+}}$activation threshold in presence of the $\gamma$-dithiol [36], yielding half-maximal rates $\sim 0.7 \Delta \mathrm{pH}$ units apart.

The knowledge accumulated through in vitro studies has not yet been confirmed in vivo, which is the main aim of this work. Because the $\mathrm{CF}_{1} \mathrm{~F}_{\mathrm{o}}$ activity is both $\Delta \tilde{\mu}_{\mathrm{H}^{+}}$and redox regulated, the study of its redox regulation requires the in vivo measurement of both $\mathrm{CF}_{1} \mathrm{~F}_{\mathrm{o}}$ activity and $\Delta \tilde{\mu}_{\mathrm{H}^{+}}$extent. The first can be measured via routinely used techniques. Since photosynthetic membranes are spectroscopic voltmeters, the $\Delta \Psi$ can be probed linearly by ECS, the electrochromic shift of photosynthetic pigments [37, 38, reviewed in 39]. Following a light-induced membrane energization in dark-adapted samples, the $\Delta \Psi$ (and ECS) decay is tightly linked to $\mathrm{H}^{+}$translocation via $\mathrm{CF}_{1} \mathrm{~F}_{\mathrm{o}}$. ECS decay kinetics following a single-turnover laser flash are therefore commonly used to probe $\mathrm{CF}_{1} \mathrm{~F}_{\mathrm{o}}$ activity in vitro [e.g., Ref. 11] as well as in vivo [e.g., Refs. 33, 40]. In contrast, the measurement of the $\Delta \tilde{\mu}_{\mathrm{H}^{+}}$is a methodological bottleneck but a protocol was proposed previously [16] whose principle can be explained through a simple analogy. The water volume contained in an opaque bottle cannot be read, but its empty volume can be easily measured as the volume of water that needs to be added before it spills out (when the maximal countenance capacity is reached). Even if the maximal countenance capacity of the bottle remains unknown, the water volume can be given in relation to this reference/leak value. The same applies for the measurement of the electrochemical proton gradient in the dark. Joliot and Joliot have shown that a threshold of electrochemical proton gradient exists, corresponding to the leak of protons through the thylakoid membrane in vivo $\left(\Delta \tilde{\mu}_{\mathrm{H}^{+} \text {leak }}\right)$. It is identified as the maximal ECS that can be sustained by a short illumination with very strong light. The $\Delta \tilde{\mu}_{\mathrm{H}^{+} \text {leak }}$ is a constant in a given photosynthetic material and can therefore be used as a "maximal countenance capacity" reference [16]. In brief, the ECS-based protocol to measure the extent of $\Delta \tilde{\mu}_{\mathrm{H}^{+} \text {dark }}$ consists in energizing the membrane with a very strong light pulse and probing the ECS increase until the $\Delta \tilde{\mu}_{\mathrm{H}^{+} \text {leak }}$ is reached (i.e., measuring the "empty volume"). This method was used previously with success [16], but some of the implicit hypothesis of this method deserved to be further tested: (i) The increase of ECS (which probes $\Delta \Psi$ ) reflects the increase of $\Delta \tilde{\mu}_{\mathrm{H}^{+}}$or, in other words, there is no significant $\Delta \mathrm{pH}$ generation during the short saturating pulse. Moreover, since the saturating pulse surpasses $\Delta \tilde{\mu}_{\mathrm{H}^{+} \text {leak, }}$ the leak process in vivo can either occur through $\Delta \tilde{\mu}_{\mathrm{H}^{+-}}$-regulated $\mathrm{H}^{+}$channels or via $\mathrm{CF}_{1} \mathrm{~F}_{\mathrm{o}}$. (ii) The method, tested only on dark-adapted leaves, remains valid whatever the redox state of the $\gamma$-subunit domain. In this work, we demonstrate that those hypotheses are respected making this method valid to assess the $\Delta \tilde{\mu}_{\mathrm{H}^{+}}$in both the reduced and oxidized states of $\mathrm{CF}_{1} \mathrm{~F}_{\mathrm{o}}$. This allowed us to confirm that $\mathrm{CF}_{1} \mathrm{~F}_{\mathrm{o}}$ is mostly in the oxidized state in dark-adapted leaves (in contrast with a previous report [16]) and to validate in vivo the model of Junesch and Gräber. Our results show that the $\Delta \tilde{\mu}_{\mathrm{H}^{+}}$activation of the disulfide-containing $\mathrm{CF}_{1} \mathrm{~F}_{\mathrm{o}}$ was $\sim 45 \mathrm{mV}$ lower and, based on the $\mathrm{CF}_{1} \mathrm{~F}_{\mathrm{o}}$ activity profile in leaves infiltrated with a thiol agent, the efficiency of the chemical reduction of the $\gamma$-subunit was a little less than $50 \%$. Finally, as a further improvement of the method, we first utilized the fact that the $\Delta \tilde{\mu}_{\mathrm{H}^{+}}$is in equilibrium with the $[A T P] /([A D P][P])$ ratio to follow the lifetime of ATP after a light perturbation, and second determined the $\Delta \tilde{\mu}_{\mathrm{H}^{+}}$partitioning into the $\Delta \mathrm{pH}$ and $\Delta \Psi$ components when incrementing illumination time. 
Material and Methods

Absorption changes. The experimental setup was as described previously [16]. Absorption changes were measured using a JTS spectrophotometer (Biologic). Pulses of saturating light are provided by LEDs peaking at $630 \mathrm{~nm}\left(\sim 2500 \mu \mathrm{mol}\right.$ photons $\left.\mathrm{m}^{-2} \mathrm{~s}^{-1}\right)$, unless otherwise stated. This light irradiance corresponds to $\sim 2000$ photons absorbed per second per PSI or per PSII, based on the measurement of changes in the membrane potential $(\Delta \Psi)$ according to the method described previously [16].

Correction and normalization of ECS signals. The light-induced membrane potential changes $(\Delta \Delta \Psi)$ were measured through the absorption changes at $520 \mathrm{~nm}$ (reflecting mostly ECS) for experiments with short pulses (less than $20 \mathrm{~ms}$ ), and $520 \mathrm{~nm}-546 \mathrm{~nm}$ for longer pulses (Fig 2). Indeed, subtracting $546 \mathrm{~nm}$ allows to correct for the contribution of cytochromes, P700 and scattering. However, the comparison between corrected data (520-546) and uncorrected data (520) shows that the correction was not necessary for short pulses (Fig S1). To express the measured ECS signals in charge separations per photosystem I (PSI), we calibrated the ECS signal by the ECS increase corresponding to one charge separation per PSI. For that, we divided all measured ECS signals in this work by $1 / 2$ the ECS increase following a single-turnover flash, provided by a dye laser at $690 \mathrm{~nm}$ pumped with a Nd:Yag laser. Since all photosystems will generate one charge separation upon such a flash, the ECS measured $250 \mu$ s after the flash is proportional to the number of active PSI + PSII. Assuming equal concentrations of both photosystem reaction centers, half this signal is equal to the sole contribution of PSI.

Reproducibility. The kinetics of ECS rise and decay were reproducible between different leaves of different batches, but it was not the case of the extent of the ECS. This is because its amplitude depends on the density of reaction centers and ECS probes per leaf area, which slightly varied in different cultures. For this reason, for each experiment where the amplitude of ECS was probed, we compared samples from the same batch of culture.

Chemicals and Inhibitors. We used chemical compounds by vacuum infiltration since the method did not interfere with the $\Delta \Psi$ increase and decay kinetics as compared to non-infiltrated samples (see Fig S2). All chemicals were purchased from Sigma-Aldrich. Tris(2-carboxyethyl)phosphine hydrochloride (TCEP) was dissolved in water at $0.3 \mathrm{M}$ and aliquots, adjusted to $\mathrm{pH} 7.0$, were frozen until further use. Solutions of antimycin-A (AA, $40 \mathrm{mM})$, nonactin $(40 \mathrm{mM})$, nigericin (Nig, $10 \mathrm{mM}$ ) and Carbonyl cyanide 4-(trifluoromethoxy)phenylhydrazone $(1 \mathrm{mM})$ were dissolved in ethanol and usually served as $1000 \mathrm{x}$ stocks that were stored at $-20^{\circ} \mathrm{C}$. 
Results

\section{Membrane potential decay measurements after saturating light pulses.}

In order to quantify the $\mathrm{CF}_{1} \mathrm{~F}_{\mathrm{o}}$ performance in $\gamma$-disulfide- and $\gamma$-dithiol-promoting conditions, we will first elaborate on an improved protocol which is based on a previous study [16] and allows to measure the $\Delta \tilde{\mu}_{\mathrm{H}^{+}}$already present in dark-adapted leaves $\left(\Delta \tilde{\mu}_{\mathrm{H}^{+} \text {dark }}\right)$. The method is based on the kinetics of the ECS and measures the $\Delta \Psi$ changes $(\Delta \Delta \Psi)$ induced by a short saturating light pulse, when compared to the baseline $\Delta \Psi$ prior to the pulse (Fig 1). We make the reasonable hypothesis that short pulses $(<20 \mathrm{~ms})$ induce exclusively $\Delta \Delta \Psi$ and that changes in the $\Delta \mathrm{pH}$ can be neglected [16] due to the high buffering capacity inside the lumen [41]. According to this hypothesis (tested in section 2), ECS changes which are strictly speaking proportional to $\Delta \Delta \Psi$, equally reflect changes in the electrochemical proton gradient $\left(\Delta \Delta \tilde{\mu}_{\mathrm{H}^{+}}\right)$. As explained before [16], since the $\mathrm{CF}_{1} \mathrm{~F}_{\mathrm{o}}$ is reversible and outpaces slow passive ion movements across the membrane, the $\Delta \tilde{\mu}_{\mathrm{H}^{+}}$dark and the [ATP]/([ADP][P]) ratio reach a thermodynamic equilibrium in dark-adapted leaves, which make the $\Delta \tilde{\mu}_{\mathrm{H}^{+}}$dark dependent on the physiological conditions. Indeed, thanks to an ancient nucleotide import machinery, the chloroplast ATP level is itself strictly dependent on the metabolic coupling with mitochondria $[16,42-$ 46].

Fig $1 \mathrm{~A}$ illustrates the influence of the baseline $\Delta \tilde{\mu}_{\mathrm{H}^{+} \text {dark }}$ prior to the pulse on the amplitude and kinetics of the pulse-induced ECS (or $\Delta \Delta \tilde{\mu}_{\mathrm{H}^{+}}$). WT leaves were infiltrated with water and a low [ATP]/([ADP][P]) ratio was obtained under impairment of the mitochondrial respiration upon the addition of antimycinA (AA), an inhibitor of the cytochrome bc1 (open symbols in Fig 1). On the contrary, high $[A T P] /([A D P][P])$ ratios were reached after a 30-s illumination (closed symbols). In all curves of Fig $1 \mathrm{~A}$, the ECS reached a plateau during the pulse $\left(\Delta \tilde{\mu}_{\mathrm{H}^{+} \text {leak }}\right)$ followed by a fast decay phase. Both the plateau and the amplitude of the fast phase depend on the light intensity (Fig S3). Such a fast phase has been observed in spinach [16] and is attributed to $\mathrm{H}^{+}$leaks that are triggered above a critical $\Delta \tilde{\mu}_{\mathrm{H}^{+}}$potential $\left(\Delta \tilde{\mu}_{\mathrm{H}^{+} \text {leak }}\right)$, which is an absolute reference of the electrochemical proton gradient in a given material in vivo. However, after $10 \mathrm{~ms}$ decay, the ECS decay kinetics becomes independent of the light intensity (the leak process contributions is over at this time, Fig S3) and reflect the $\mathrm{H}^{+}$release from the lumen via $\mathrm{CF}_{1} \mathrm{~F}_{0}$ that is associated with ATP synthesis [16]. The ECS reached $10 \mathrm{~ms}$ after the end of the pulse (ECS $10 \mathrm{~ms}$ ) is therefore independent of pulse intensity (see Fig S3), and also independent of the physiological conditions preceding the pulse [16]. Thus, the ECS $10 \mathrm{~ms}$ will be used hereafter as the experimental reference of $\Delta \tilde{\mu}_{\mathrm{H}^{+}}$and ECS data in the following will appear in negative values (ECS $\mathrm{ECS}_{10 \mathrm{~ms}} ; \mathrm{Fig} 1 \mathrm{~B}$ ) and correspond to the "empty volume" of the opaque bottle analogy. The light intensity of the pulse will be kept constant throughout the study (see Material and Methods).

The amplitude of the ECS increase was higher in the presence of AA than after the pre-illumination (Fig $1 A)$. Given that the reached $\Delta \tilde{\mu}_{\mathrm{H}^{+} \text {leak }}$ at the end of the pulse is an absolute reference of $\Delta \tilde{\mu}_{\mathrm{H}^{+}}$, the higher pulse-induced ECS in the presence of AA did not reflect a higher $\Delta \tilde{\mu}_{\mathrm{H}^{+}}$at the end of the pulse in those conditions. It rather reflected a lower baseline $\Delta \tilde{\mu}_{\mathrm{H}^{+} \text {dark }}$ before the pulse, which was revealed after rescaling to our reference $\mathrm{ECS}_{10 \mathrm{~ms}}$ (-6.7 vs. >-3.5 charge separations/PSI, Fig 1B). The ECS amplitudes during the pulse depended on the $\Delta \tilde{\mu}_{\mathrm{H}^{+} \text {dark }}$ which is a pedestal onto which an extra $\Delta \tilde{\mu}_{\mathrm{H}^{+}}$ is superimposed until the total $\Delta \tilde{\mu}_{\mathrm{H}^{+}}$reaches $\Delta \tilde{\mu}_{\mathrm{H}^{+} \text {leak }}[16]$. After the 30-s illumination, the increase of the amplitude of the pulse with the time in darkness (Fig $1 \mathrm{~A}$ ), again, indicated the continuous decrease 
of the $\Delta \tilde{\mu}_{\mathrm{H}^{+} \text {dark }}$ (Fig 1B) and reflected the consumption of the extra ATP produced during the 30-s illumination. According to Fig $1 \mathrm{~B}$ and a previous report [16], the ECS decay kinetics after the pulse displayed three phases. A first phase, completed in $10 \mathrm{~ms}$, was associated with $\mathrm{H}^{+}$leaks (Fig $1 \mathrm{~A}$ ) as discussed before and will be discarded from now on. A second phase was completed in $100 \mathrm{~ms}$ (phase 2 ) and was linked to ATP synthesis. Then, a multiphasic slow decay (phase 3 ), completed in $~ 1$ minute, was observed only once the $\Delta \tilde{\mu}_{\mathrm{H}^{+}}$fell below a threshold (at the end of phase 2, $\sim 100 \mathrm{~ms}$ ). This threshold corresponds to the $\Delta \tilde{\mu}_{\mathrm{H}^{+}}$activation described before [11-16] and represented a second reference level [15], about 2 charge separations/PSI below ECS 10ms (arrowhead in Fig 1B). Phase 3 indicated a slowly active $\mathrm{CF}_{1} \mathrm{~F}_{0}$, or, alternatively, a small $\mathrm{CF}_{1} \mathrm{~F}_{0}$ fraction staying fully active.

We first decided to check whether this quantitative measurement of the $\Delta \tilde{\mu}_{\mathrm{H}^{+} \text {dark }}$ remained valid regardless of the redox regulation mechanism. Figs $1 \mathrm{C}$ and $1 \mathrm{D}$ show similar experiments on leaves infiltrated with a thiol reductant, TCEP. Furthermore, the samples were in the same physiological conditions as in Figs 1A/B (AA-treated or pre-illuminated samples). In all cases, the ECS generation and the decay in the first tens of $\mathrm{ms}$ after the end of the pulse resembled the untreated sample. As in water-infiltrated leaves, AA-treated leaves in the presence of TCEP showed a lower $\Delta \tilde{\mu}_{\mathrm{H}^{+}}$dark and illumination increased this pedestal (dashed lines in Fig 1D), reflecting ATP accumulation during preillumination. The $\Delta \tilde{\mu}_{\mathrm{H}^{+}}$decay kinetics were always monophasic; we did not observe the slow phase of ECS decay (absence of phase 3 in Fig 1D). This is true even in conditions where $\Delta \tilde{\mu}_{\mathrm{H}^{+} \text {dark }}$ was below the $\Delta \tilde{\mu}_{\mathrm{H}^{+}}$activation of Fig $1 \mathrm{~B}$, i.e. when dark re-adaptation after the 30-s illumination was longer than 9 min or in AA-treated samples. This observation was in agreement with the higher $\mathrm{CF}_{1} \mathrm{~F}_{\mathrm{o}}$ activity measured in vitro upon cleaving the $\gamma$-disulfide by chemical thiol reductants, at low $\Delta \tilde{\mu}_{\mathrm{H}^{+}}$only [36]. A quantitative analysis will be given in section 4 , demonstrating how the effect of the $\gamma$-redox state influences the $\Delta \tilde{\mu}_{\mathrm{H}^{+}}$dependence of $\mathrm{CF}_{1} \mathrm{~F}_{\mathrm{o}}$ activity.
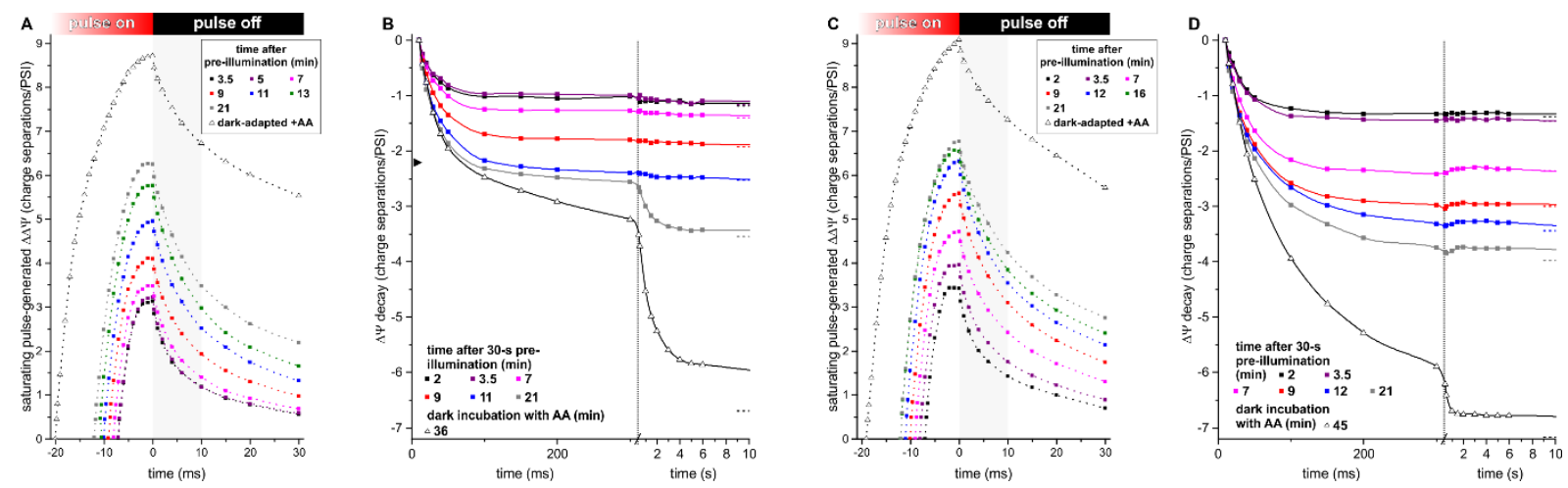

Fig 1: Pulse-induced ECS kinetics are shown in water-infiltrated leaves in the absence (A-B) or presence (C-D) of the mild reductant TCEP at $50 \mathrm{mM}$. Dark-adapted leaves were illuminated for $30 \mathrm{~s}$ (green-orange LED, $1000 \mu \mathrm{mol}$ photons $\mathrm{m}^{-2} \mathrm{~s}^{-1}$ ), followed by a dark re-adaptation for several minutes (indicated in the legend). +AA: infiltrated with $40 \mu \mathrm{M}$ antimycin-A without illumination. All the measurements were made on different leaves from the same plant. In panels A and C, the dark-adapted (baseline) ECS level was arbitrarily set to 0 and only the ECS

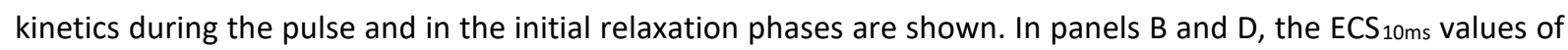
all curves were set to 0 and only the ECS relaxation in the dark is shown (the value reached after 1 min relaxation is indicated with three dots on the right). The transition between the fast and slow relaxation phases $\left(\Delta \tilde{\mu}_{\mathrm{H}^{+}}\right.$activation $)$is indicated in panel $\mathrm{B}$ by an arrowhead. 


\section{Relative extent of $\Delta \Psi$ and $\Delta p H$ induced by short light pulses.}

For the interpretation of the previous results regarding the evolution of $\Delta \tilde{\mu}_{\mathrm{H}^{+}}$, we followed the opaque bottle analogy and have made the hypothesis that the experimental reference of the short saturating light pulses, ECS ${ }_{10 m s}$, served as our $\Delta \tilde{\mu}_{\mathrm{H}^{+}}$reference. This implied that the $\Delta \mathrm{pH}$ that was generated until reaching $\mathrm{ECS}_{10 \mathrm{~ms}}$ was insignificant, and only $\Delta \Psi$ increased during the short saturating light pulses. To test our hypothesis, we first obtained a non-perturbed ECS $_{10 m s}$ reference from dark-adapted (for several minutes) leaves by applying a short 12-ms probing pulse (dark line, Fig $2 \mathrm{~A}$ ). Then, we applied saturating light pulses of variable duration to the dark-adapted samples and probed, after a relatively short dark period of $15 \mathrm{~s}$, the extra $\Delta \Psi(\Delta \Delta \Psi)$ that was sustained. The $\Delta \Psi$ was monitored throughout the measurement and $\Delta \Delta \Psi$ was calculated as the difference between the dark-adapted ECS signal before the first light perturbation and the ECS signal 15-s later (Fig 2A). The extra $\Delta \tilde{\mu}_{\mathrm{H}^{+}}\left(\Delta \Delta \tilde{\mu}_{\mathrm{H}^{+}}\right)$that was present 15-s after the first light perturbation was obtained, again, from a 12-ms probing pulse (i.e., from $\left.\mathrm{ECS}_{10 \mathrm{~ms}}\right)$. Especially after prolonged light pulses, the $\mathrm{ECS}_{10 \mathrm{~ms}}$ was below the non-perturbed ECS $S_{10 m s}$ from dark-adapted material. We attributed this lowering of our electrogenic $\Delta \tilde{\mu}_{\mathrm{H}^{+}}$reference to the extra $\Delta \mathrm{pH}(\Delta \Delta \mathrm{pH})$ that was present after $15 \mathrm{~s}$ dark. A duration of $15 \mathrm{~s}$ dark was necessary in this experiment to allow a partial relaxation of the electron carriers toward the dark-adapted state. Indeed, at the end of the first light pulse, the photochemistry of both photosystems was low due to the absence of acceptors (oxidized plastoquinones or oxidized ferredoxins) or donors (reduced P700). If the second light pulse was applied too early, photochemistry was insufficient to reach the $\Delta \tilde{\mu}_{\mathrm{H}^{+} \text {leak }}$ which made measurement of the $\Delta \tilde{\mu}_{\mathrm{H}^{+}}$impossible.

We followed those parameters in water-infiltrated and in nigericin-treated leaves for different durations of the first pulse (10 ms to $1 \mathrm{~s}$ ), given $15 \mathrm{~s}$ before the probing 12-ms pulse. For every first pulse duration, the ECS $10 \mathrm{~ms}$ upon the second pulse was smaller than in the corresponding dark-adapted sample (Fig 2B). This indicated that a significant amount of extra ATP, generated during and after the first illumination, was still present after $15 \mathrm{~s}$ dark and increased of the $\Delta \tilde{\mu}_{\mathrm{H}^{+}}$(more precisely, $\Delta \Delta \mathrm{pH}$ ) compared to the dark-adapted level. There was a significant change in the lowered ECS ${ }_{10 \mathrm{~ms}}$ between the 100-ms and 500-ms light perturbation. In water-infiltrated samples, the $\Delta \Delta \Psi$ at $15 \mathrm{~s}$ after the first perturbation (dashed line in Fig 2B) was significant, up to 0.4 charge separation/PSI. The $\Delta \Delta \Psi$ appeared to stagnate upon light perturbations longer than 100-ms.

We used the ionophore nigericin (Nig), which exchanges protons with $\mathrm{K}^{+}$, and it is important to note that Nig also collapsed the proton motive force for mitochondrial ATP synthesis and thus interfered with metabolic coupling between the organelles. Accordingly, Nig infiltration resulted in a collapse of $\Delta \tilde{\mu}_{\mathrm{H}^{+} \text {dark }}$ in the chloroplast (within $\sim 15-30$ min incubation, see Fig S4 and discussion). Here, Nig was used as a proof of principle to favor $\Delta \Delta \Psi$ at the expense of $\Delta \Delta \mathrm{pH}$. Nig-infiltrated samples were analyzed in Fig $2 \mathrm{C}$ and the extent of the lowered $\mathrm{ECS}_{10 \mathrm{~ms}}$ as a function of the first light pulse duration $(\Delta \Delta \mathrm{pH})$ showed similar developments as the controls in Fig 2B. However, the $\Delta \Delta \Psi$ after $15 \mathrm{~s}$ was always higher than 1 charge separation/PSI, even for the shortest durations of the light pulse (dashed line in Fig $2 \mathrm{C}$ ). The probed $\Delta \Delta \tilde{\mu}_{\mathrm{H}^{+}}$stored $15 \mathrm{~s}$ after a 10 -ms pulse was 0.4 and 1.4 charge separations/PSI in control and Nig samples, respectively. For a 1-s pulse, those values reached 1.1 and 2.8 charge separations/PSI for control and Nig samples, respectively. In water-infiltrated samples (Fig 2D), the dependency of $\Delta \Delta \mathrm{pH}$ and $\Delta \Delta \Psi$ with respect to the first pulse duration indicated that short light pulses (100-ms or below) induced mostly $\Delta \Delta \Psi$ whereas $\Delta \Delta \mathrm{pH}$ dominated after longer light pulses 
(500-ms or 1-s). In contrast (Fig 2E), presence of Nig promoted a very strong $\Delta \Delta \Psi$ which was present $15 \mathrm{~s}$ after the first light pulse, whereas the amplitude of the $\Delta \Delta \mathrm{pH}$ was a lot smaller regardless of the pulse duration.

The fact that short pulses (below $100 \mathrm{~ms}$ ) generated only small changes in $\Delta \mathrm{pH}$ validated our initial hypothesis. The extent of $\Delta \mathrm{pH}$ was smaller than 0.1 charge separation/PSI. This indicated that the ECS pulse method allowed the quantitative measurement of $\Delta \tilde{\mu}_{\mathrm{H}^{+}}$with a potential error of 0.1 charge separation/PSI only. The higher $\Delta \tilde{\mu}_{\mathrm{H}^{+}}$storage after $15 \mathrm{~s}$ dark in the Nig samples was expected, simply because the relaxation of the pulse-induced $\Delta \tilde{\mu}_{\mathrm{H}^{+}}$was slower when the $\Delta \tilde{\mu}_{\mathrm{H}^{+} \text {dark }}$ was below the $\Delta \tilde{\mu}_{\mathrm{H}^{+}}$activation, as we described before in the case of a AA treated sample (Fig 1B). Compared to AA, the uncoupling by Nig was more efficient in collapsing $\Delta \tilde{\mu}_{\mathrm{H}^{+} \text {dark }}$ but, like other uncouplers, it modified the different phases of ECS decay kinetics (Fig S4, see also discussion). Therefore, the use of AA will be preferred instead of Nig in the following sections when collapsing the $\Delta \tilde{\mu}_{\mathrm{H}^{+}}$dark will be needed, because AA preserves membrane integrity. 

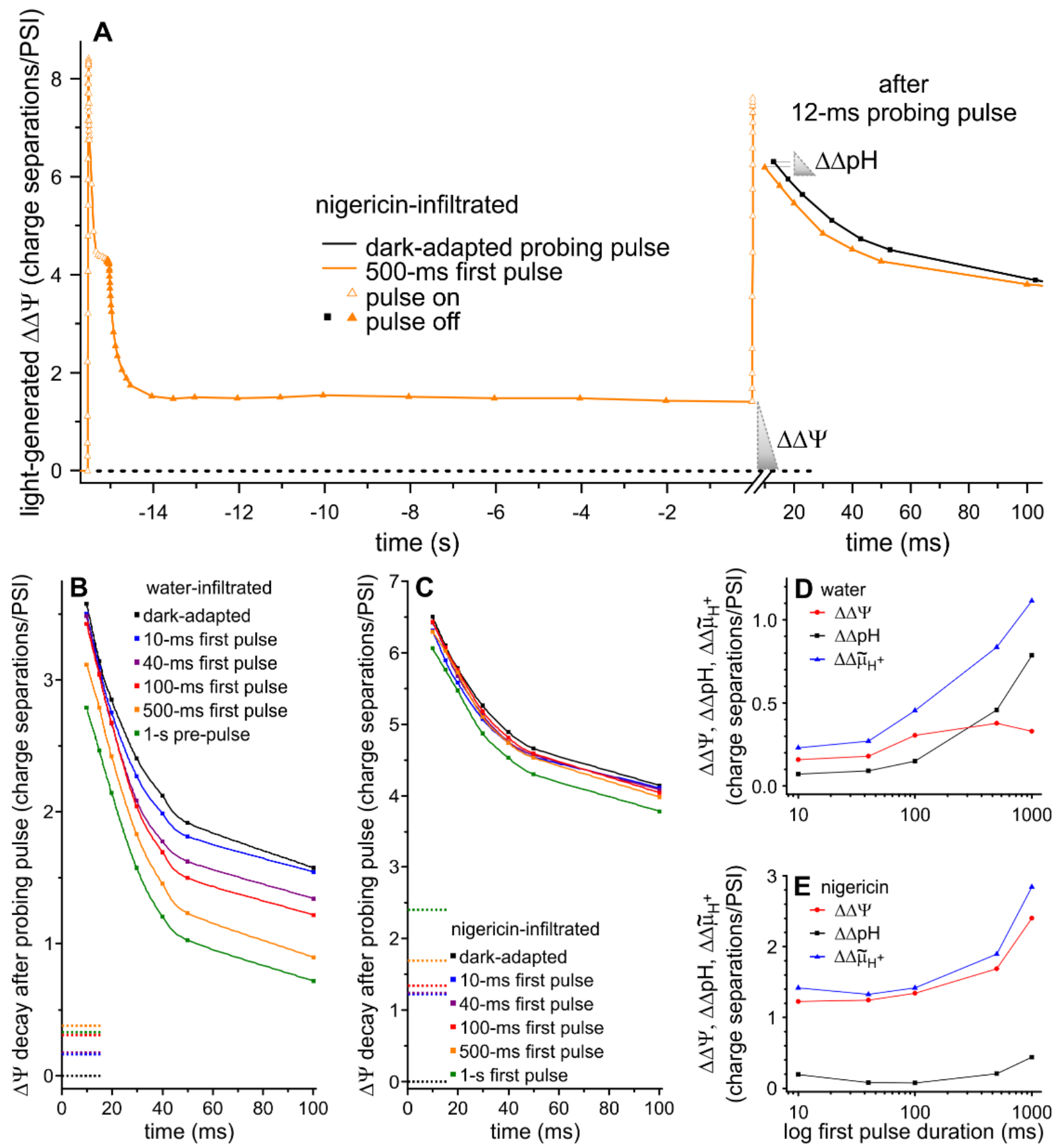

Fig 2: The estimation of $\Delta \Delta \Psi, \Delta \Delta \mathrm{pH}$ and $\Delta \Delta \tilde{\mu}_{H^{+}}$generated by an illumination is shown. The parameters were measured $15 \mathrm{~s}$ after a saturating pulse of various duration. (A) Exemplary ECS measurement in the presence of nigericin (open symbols light on, closed symbols darkness) measured as the difference between the absorption changes at $520 \mathrm{~nm}$ and the one at $546 \mathrm{~nm}$ (see Methods). The leaf was illuminated with a first pulse of 500-ms $(t=-15 \mathrm{~s})$, then ECS was followed for $15 \mathrm{~s}$ in the dark and a 12-ms probing pulse was used to determine the new $\mathrm{ECS}_{10 \mathrm{~ms}}$ reference (the probing pulse ended at $t=0 \mathrm{~s}$ ). For the sake of visualization, the dark-adapted ECS decay was shifted on the $x$-axis by $3 \mathrm{~ms}$. (B) and (C) The initial phase of the $\Delta \Psi$ decay after the 12 -ms pulse is shown for leaves infiltrated with (B) water and (C) nigericin. The $\Delta \Delta \Psi$ at the end of the 15 -s dark period is indicated by horizontal dashed lines. The $\Delta \Delta \mathrm{pH}$ corresponds to the difference between the $\mathrm{ECS}_{10 \mathrm{~ms}}$ after the saturating pulse and the one of a dark-adapted leaf (dark closed squares). (D) and (E) The relationship of $\Delta \Delta \Psi, \Delta \Delta \mathrm{pH}$ and $\Delta \Delta \tilde{\mu}_{H^{+}}$ with the duration of the first light pulse are shown for water (D) and nigericin (E) infiltrated leaves. All the measurements in this figure were performed with leaves from the same plant. 


\section{What is the $\Delta \tilde{\mu}_{\mathrm{H}^{+}} /$ATP lifetime after an illumination?}

In the previous sections, we have shown that our methodology allows quantitative measurements of the $\Delta \tilde{\mu}_{\mathrm{H}^{+} \text {dark }}$ (relative to the $\Delta \tilde{\mu}_{\mathrm{H}^{+}}$reference given by $\mathrm{ECS}_{10 \mathrm{~ms}}$ ) provided that the saturating light pulse is (i) strong enough to reach the $\Delta \tilde{\mu}_{\mathrm{H}^{+} \text {leak }}$ (which is valid for the 2000 photons/PSI/s light irradiance used here) and (ii) short enough to avoid the generation of $\Delta \mathrm{pH}$ (which is valid for pulses shorter than $100 \mathrm{~ms})$. In this section, we use this method to follow the fate of the $\Delta \tilde{\mu}_{\mathrm{H}^{+}}$after an illumination by supposing that the thermodynamic equilibrium is met during a dark period before the ECS measurement and, as discussed before, following $\Delta \tilde{\mu}_{\mathrm{H}^{+}}$is equivalent to following the [ATP]/([ADP][P]) ratio. In Fig 1B, we measured the $\Delta \tilde{\mu}_{\mathrm{H}^{+}}$dark at different time in darkness following a 30-s illumination in water-infiltrated leaves. In Fig $3 \mathrm{~A}$, we reported the values of the $\Delta \tilde{\mu}_{\mathrm{H}^{+}}$dark as a function of the time in darkness. In the water-infiltrated leaf, the $\Delta \tilde{\mu}_{\mathrm{H}^{+} \text {dark }}$ after the 30-s illumination was $\sim-1.2$ charge separations/PSI relatively to the $\Delta \tilde{\mu}_{\mathrm{H}^{+}}$reference (ECS $\left.{ }_{10 \mathrm{~ms}}\right)$, a value significantly higher than the darkadapted one ( $\sim-4$ charge separations/PSI, black dashed line). This value remained constant for 5 minutes following the illumination and, after this lag, relaxed to the dark-adapted value in $\sim 30$ minutes (Fig 3A). As discussed before, this reflected the build-up of ATP during illumination, and the subsequent slow consumption of this extra ATP. This consumption may be associated with slow passive $\mathrm{H}^{+}$leaks through the membrane and/or with ATP-consuming reactions occurring in the chloroplast stroma. When leaves were infiltrated with Nig and illuminated for $30 \mathrm{~s}$ (Fig 3A), the $\Delta \tilde{\mu}_{\mathrm{H}^{+} \text {dark }}$ reached a lower level (-2.3 charge separations/PSI after 2 min dark) and relaxed to an even lower value within 15 minutes (-6.3 charge separations/PSI). This low value in a dark-adapted sample reflected the fact that the $\Delta \tilde{\mu}_{\mathrm{H}^{+}}$dark is suppressed due to the absence of mitochondrial ATP production in darkness, as discussed before. The ATP generated in the chloroplast during the illumination might be consumed more rapidly in the presence of Nig, probably because the metabolic needs cannot be met without mitochondrial ATP production.

Fig $3 \mathrm{~A}$ also shows the lifetime of $\Delta \tilde{\mu}_{\mathrm{H}^{+} \text {dark }}$ and ATP after illuminations of different durations (between 3-s and 12-min) in non-infiltrated leaves of the WT. Interestingly, if the duration of the illumination reached 7-s or more, the $\Delta \tilde{\mu}_{\mathrm{H}^{+} \text {dark }}$ attained at the end of the illumination period became independent on the illumination (between $\sim-1.3$ to -1.5 charge separations/PSI). However, a lag was observed before the $\Delta \tilde{\mu}_{\mathrm{H}^{+} \text {dark }}$ started to decay; whose duration increased with the time of illumination. Then, ATP levels decreased continuously after illumination and reached in $\sim 20 \mathrm{~min}$ a stable dark level corresponding to the dark-adapted value ( $\sim 3.5$ charge separations/PSI). In this view, the slow decrease of $\Delta \tilde{\mu}_{\mathrm{H}^{+} \text {dark }}$ reflected the consumption of the ATP produced during the illumination or of the accumulated high energy bonds in different plastid metabolites, to which the ATP pool is buffered (see Discussion/Conclusion). The water-infiltration does not seem to have an impact on the behavior of the leaf, at least for illuminations not longer than $30 \mathrm{~s}$. This is illustrated by the fact that the curve described before (30-s illumination of a water-infiltrated leaf) stands in between the 15-s and 40-s illumination curves for non-infiltrated leaves (Fig 3A). However, the behavior of the water-infiltrated leaves differ from the non-infiltrated ones for illuminations longer than $30 \mathrm{~s}$, which might be due to the lower $\mathrm{CO}_{2}$ availability in water-infiltrated leaves, and therefore a lower activation of the Calvin-Benson cycle.

The final goal of this manuscript will be to establish the $\Delta \tilde{\mu}_{\mathrm{H}^{+}}$dependency of the $\mathrm{CF}_{1} \mathrm{~F}_{\mathrm{o}}$ activity in the reduced and oxidized forms. Accordingly, we compared leaves infiltrated with water or with TECP, which promotes $\gamma$-disulfide cleavage. We also looked at the behavior of the gamera mutant, in which the $\mathrm{CF}_{1} \mathrm{~F}_{\mathrm{o}}$ is in its reduced form ( $\gamma$-dithiol) in the dark [33]. The $\Delta \tilde{\mu}_{\mathrm{H}^{+}}$threshold $\left(\Delta \tilde{\mu}_{\mathrm{H}^{+}}\right.$activation $)$at $\sim 100$ 
$m s$ in darkness, which is characteristic for water-infiltrated samples with the $\gamma$-disulfide (arrowhead in Fig 3B, see also Fig 1B), disappeared in presence of the TCEP due to accelerated $\Delta \tilde{\mu}_{\mathrm{H}^{+}}$decay kinetics at the end of phase 2. Like in TCEP-infiltrated WT, the $\Delta \tilde{\mu}_{\mathrm{H}^{+}}$decay kinetics in the gamera mutant was accelerated and was even faster. In both cases, the $\Delta \tilde{\mu}_{\mathrm{H}^{+}}$decay kinetics was completed in $\sim 1 \mathrm{~s}$. The faster decay kinetics in the gamera mutant, obtained in the dpa1 genetic background [33], could be unambiguously attributed to the presence of the dithiol since the behavior of the ATPC1complemented dpa1 mutant, dpa1c, was similar to the WT (Fig 3B; ATPC1 is the thioredoxin-sensitive $\gamma$-subunit isoform and ATPC2 is the isoform present in gamera). The difference of ECS decay between gamera and TCEP-infiltrated WT leaves suggests that TCEP did only partially reduce the disulfide bond in the dark. Limited efficiency of TCEP in cleaving protein disulfides has been described elsewhere [47]. Based on the pronounced slowdown of the $\Delta \tilde{\mu}_{\mathrm{H}^{+}}$decay after phase 2 , we conclude that $\mathrm{CF}_{1} \mathrm{~F}_{\mathrm{o}}$ was present mainly in its oxidized state in dark-adapted WT leaves, in contradiction with a previous report [16]. Addition of TCEP promoted the reduction of $\gamma$-subunit disulfide in a fraction of $\mathrm{CF}_{1} \mathrm{~F}_{\mathrm{o}}$ (distributed homogeneously along the thylakoid membrane), resulting in a mixture of active and inactive $\mathrm{CF}_{1} \mathrm{~F}_{\mathrm{o}}$ and a continuous decay kinetics of the $\Delta \tilde{\mu}_{\mathrm{H}^{+}}$.

In TCEP-treated leaves, the $\Delta \tilde{\mu}_{\mathrm{H}^{+} \text {dark }}$ attained after the 30-s illumination was similar to the one in waterinfiltrated leaves ( -1.5 charge separations/PSI, Fig 3A), but the lag was slightly shorter leading to a shorter lifetime of the $\Delta \tilde{\mu}_{\mathrm{H}^{+} \text {dark }}$ and ATP, respectively. We cannot rule out that TCEP infiltration also influenced the redox state of some Calvin-Benson cycle enzymes, altering the ATP sink capacity in the dark. Alternatively, TCEP could also produce mild uncoupling of the membranes. It is noteworthy, however, that at variance with the effect of commonly used dithiothreitol (DTT), the kinetics of the fluorescence increase in a dark-adapted leaf was not altered upon TCEP infiltration, eliminating a potential side effect on the PQ pool redox state (not shown). In a similar experiment, we analyzed the effect of a 14-min pre-illumination on a non-infiltrated gamera mutant leaf (closed circle in Fig 3A). At variance with the results obtained in the WT, we observed that illumination of the gamera mutant induced a decrease of the $\Delta \tilde{\mu}_{\mathrm{H}^{+} \text {dark }}$ levels that returned to the dark-adapted level very slowly in $\sim 45$ min (see red horizontal line). We conclude that the mutation induced a perturbation of $\mathrm{CF}_{1} \mathrm{~F}_{\mathrm{o}}$ that prevented long term ATP accumulation during illumination. A lower gamera proton conductivity in steady-state light was reported [33] which might reflect this $\mathrm{CF}_{1} \mathrm{~F}_{\mathrm{o}}$ perturbation. The latter could be related to the large slowdown of Calvin-Benson cycle activation (about a factor 4 ) and the larger transient NPQ formation observed on the mutant with respect to the WT (see Fig S5). 

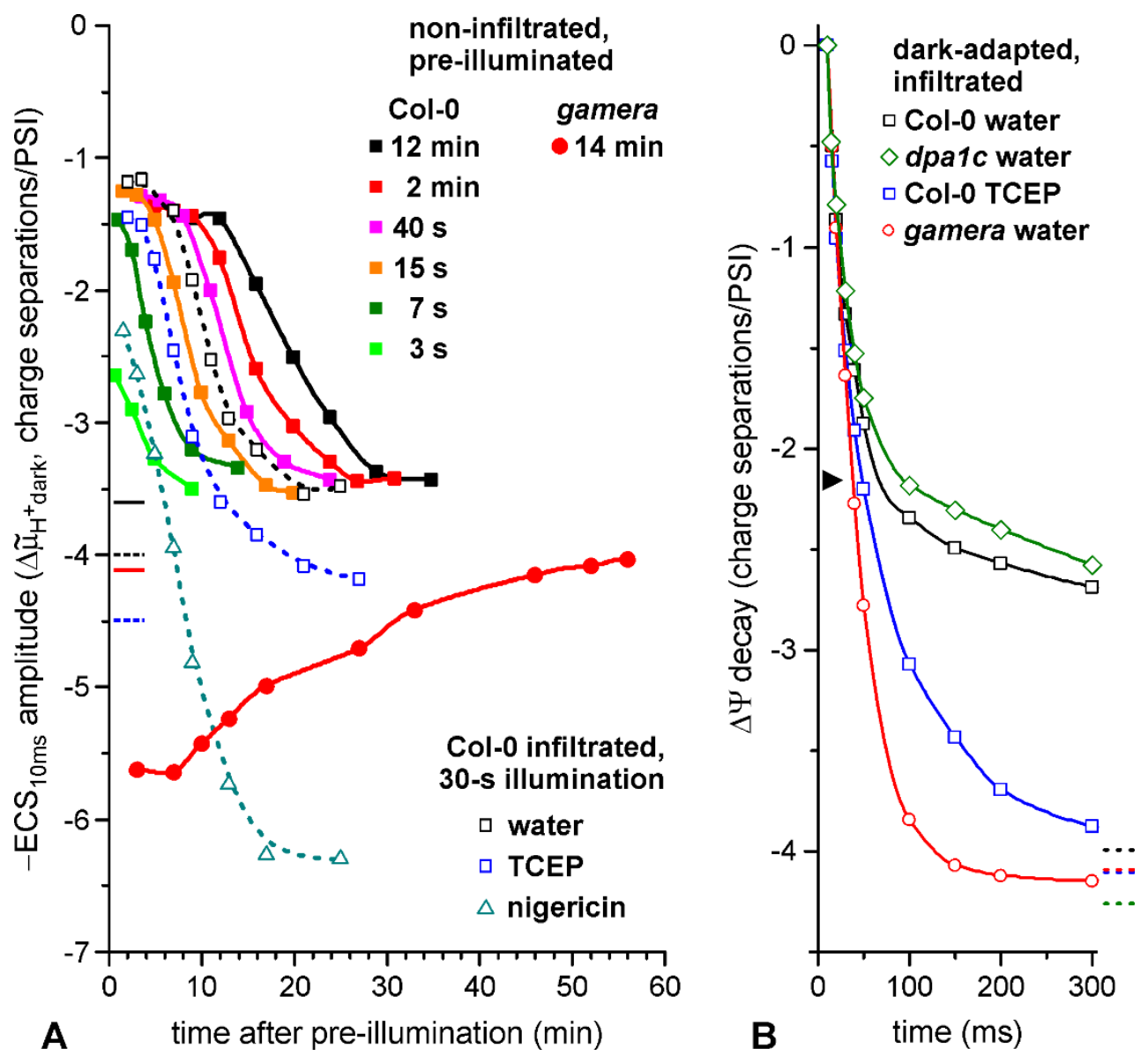

Fig 3: Light-induced ATP lifetime measurements and membrane potential $(\Delta \Psi)$ decay kinetics in various Arabidopsis genotypes are shown. (A) The effect of the $\gamma$-disulfide bond reduction on ATP accumulation and relaxation after an illumination of different durations is displayed. The $\Delta \tilde{\mu}_{\mathrm{H}^{+}}$dark values are relative to the ECS10ms and horizontal lines refer to fully dark-adapted, non-infiltrated (solid) and infiltrated (dashed) samples. The water-/TCEP-infiltrated WT samples were from the same plant, and the varied pre-illumination experiments were performed on another plant. (B) Initial $\Delta \Psi$ decay kinetics of the WT are shown (infiltrated in the dark with water or $50 \mathrm{mM}$ TCEP), as well as the gamera mutant and the ATPC1-complemented dpa1 mutant, termed dpa1c. The slowdown at $100 \mathrm{~ms}$ is shown with an arrowhead. Dashed lines indicate the $\Delta \Psi$ decay value 1 min after the pulse. 
4. $\Delta \tilde{\mu}_{H^{+}}$dependence of $\mathrm{CF}_{1} F_{o}$ rates in dependence of the $\gamma$-subunit redox state.

The final aim of this work is to determine how the reduction/oxidation of the $\gamma$-disulfide modifies the $\Delta \tilde{\mu}_{\mathrm{H}^{+}}$regulation of $\mathrm{CF}_{1} \mathrm{~F}_{\mathrm{o}}$ activity. To modulate the redox state of the $\gamma$-disulfide, we compared darkadapted water-infiltrated leaves (oxidized form) with TCEP-infiltrated leaves (partially reduced form) and the gamera mutant (fully reduced form). As discussed in the introduction, studying the $\Delta \tilde{\mu}_{\mathrm{H}^{+}}$ regulation of $\mathrm{CF}_{1} \mathrm{~F}_{\mathrm{o}}$ activity requires the in vivo measurement of both the $\Delta \tilde{\mu}_{\mathrm{H}^{+}}$and the $\mathrm{CF}_{1} \mathrm{~F}_{\mathrm{o}}$ activity. In this work, the pulse-induced ECS method to measure $\Delta \tilde{\mu}_{\mathrm{H}^{+}}$has been established and validated in both redox states. For $\mathrm{CF}_{1} \mathrm{~F}_{\mathrm{o}}$ activity determination, the ECS decay rate after a single turnover laser flash is routinely used [e.g., Ref. 40]. However, there is a bias due to the "b phase", a 10-ms ECS rise due to electrogenic contribution of the cytochrome $b_{6} f$ activity which superimposes to the $\mathrm{CF}_{1} \mathrm{~F}_{\mathrm{o}}$ activity-related ECS decay. Moreover, we observed that another source of variation occurred when using flashes, which has been documented already $[10,11]$ but remains poorly understood. We tried to circumvent these sources of error and mitigate their contributions to the ECS decay by generating the $\Delta \tilde{\mu}_{\mathrm{H}^{+}}$-activated state of $\mathrm{CF}_{1} \mathrm{~F}_{\mathrm{o}}$. The latter was routinely obtained by short saturating pulses which had a duration of at least 8-ms (and lasted up to 17-ms depending on the $\Delta \tilde{\mu}_{\mathrm{H}^{+}}$dark ; see Fig S6). We also measured the rate of $\Delta \tilde{\mu}_{\mathrm{H}^{+-}}$-activated $\mathrm{CF}_{1} \mathrm{~F}_{\mathrm{o}}$ as the slope of the ECS decay when ECS signals reached the level expected after a saturating laser flash (i.e., $\Delta \tilde{\mu}_{\mathrm{H}^{+}}$dark +1 charge separation/PSI, see Fig S6). At that time, the cytochrome $b_{6} f$ activity was mostly over and/or contributed marginally to the apparent kinetics: the $b_{6}$-catalyzed electron transfer between $\mathrm{P} 700^{+}$and $\mathrm{PQH}_{2}$ lasted longer than after a flash but this process was negligible at the time of the measurement compared to the coincident $\Delta \Psi$ consumption by $\Delta \tilde{\mu}_{\mathrm{H}^{+}}$-activated $\mathrm{CF}_{1} \mathrm{~F}_{\mathrm{o}}$.

Fig $4 \mathrm{~A}$ sums up all the $\mathrm{CF}_{1} \mathrm{~F}_{\mathrm{o}}$ rates for various $\Delta \tilde{\mu}_{\mathrm{H}^{+}}$dark values that were measured with this method. All data presented in this manuscript so far have been included, i.e. in WT leaves (dark adapted or during relaxation following a 30-s illumination), in TCEP-treated WT leaves in the same conditions, as well as in gamera (dark-adapted or following a 14-min illumination). The dark-adapted situation in the presence of AA was also added. As discussed before, the gamera mutant represents the behavior of the reduced form of the $\mathrm{CF}_{1} \mathrm{~F}_{\mathrm{o}}$ whereas the water-infiltrated $\mathrm{WT}$ represents its oxidized form and the TCEP-infiltrated WT is an intermediate situation where part of $\mathrm{CF}_{1} \mathrm{~F}_{\mathrm{o}}$ is in the oxidized form and part of $\mathrm{CF}_{1} \mathrm{~F}_{\mathrm{o}}$ is in the reduced form. Below $\Delta \tilde{\mu}_{\mathrm{H}^{+} \text {activation }}(-2.2$ charge separations/PSI, see arrow in Fig 1B, Fig $3 \mathrm{~B}$ ), the $\mathrm{CF}_{1} \mathrm{~F}_{0}$ was always faster in the reduced form (TCEP-infiltrated or gamera) than in the oxidized form (water-infiltrated). In both reduced and oxidized forms, the $\mathrm{CF}_{1} \mathrm{~F}_{\mathrm{o}}$ rate vs. $\Delta \tilde{\mu}_{\mathrm{H}^{+}}$curves resembled the ones obtained in vitro by Junesch and Gräber [36]. The rate of $\mathrm{CF}_{1} \mathrm{~F}_{\mathrm{o}}$ increased exponentially with $\Delta \tilde{\mu}_{\mathrm{H}^{+}}$in the reduced form, whereas a lag was present in the oxidized form until a specific $\Delta \tilde{\mu}_{\mathrm{H}^{+}}$activation was reached.

Both $\Delta \tilde{\mu}_{\mathrm{H}^{+}}$dark -dependent rates of (chemically unmodified) $\mathrm{CF}_{1} \mathrm{~F}_{\mathrm{o}}$ in WT and gamera have been fitted with a sigmoid function, like in [36]. In fact, the plotted $\mathrm{CF}_{1} \mathrm{~F}_{0}$ rates of both genotypes were processed in one calculation by using a sum of two sigmoid functions. In Equation 1, we forced, via the constant $c$, the first summand to be zero in the gamera plot and the second summand to be zero in the WT plot:

$y=c \times\left(\frac{A 1}{1+10^{(x 0.0 x-x) \times p}}\right)+(1-c) \times\left(\frac{A 1}{1+10^{(x 0 . r e d-x) \times p}}\right)($ Eq.1). 
$A 1$ defines shared maximal rates. With the respective standard errors, $A 1$ was $35.3 \pm 1.1 \mathrm{~s}^{-1}$. The slope parameter of the function is expressed as $p(1.4 \pm 0.1)$. The $\Delta \tilde{\mu}_{\mathrm{H}^{+}}$where the half-maximal $\mathrm{CF}_{1} \mathrm{~F}_{\mathrm{o}}$ rates were obtained for WT and gamera are defined as $\times 0.0 \times$ ( -1.5 charge separations/PSI) and $x 0$. red $(-3.8$ charge separations/PSI), respectively. The sigmoid TCEP curve in Fig $4 \mathrm{~A}$ was obtained with $c=0.56 \pm$ 0.04 , suggesting about $56 \%$ of $\mathrm{CF}_{1} \mathrm{~F}_{\mathrm{o}}$ in the oxidized form ( $\gamma$-disulfide conformation) upon infiltration with TCEP. This value was close to the fraction of reduced $\mathrm{CF}_{1} \mathrm{~F}_{0}$ in the presence of TCEP based on the kinetics of ECS decay in the gamera and WT presented in Fig 4B (already presented in Fig 3B). In Fig

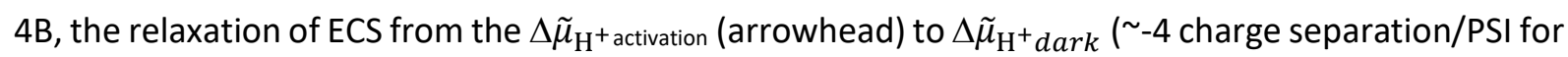
the 3 curves) was fitted with a mono-exponential decay. Rate constants of $0.8 \mathrm{~s}^{-1}$ in water-infiltrated WT were obtained in the presence of $100 \% \gamma$-disulfide, and $30.6 \mathrm{~s}^{-1}$ were measured with $100 \% \gamma$ dithiol in gamera. Accordingly, Fig $4 \mathrm{~B}$ suggests that TCEP infiltration produced $\sim 58 \% \gamma$-disulfide cleavage in $\mathrm{WT} \mathrm{CF}_{1} \mathrm{~F}_{\mathrm{o}}$, since the rate becomes $13.4 \mathrm{~s}^{-1}$.

In line with previous reports on high ATP synthesis rates under disulfide-promoting conditions [22, 36], we did not observe an effect of the redox regulation on the maximal $\mathrm{CF}_{1} \mathrm{~F}_{\mathrm{o}}$ rate at high $\Delta \tilde{\mu}_{\mathrm{H}^{+}}$in WT, even though we did not manage to reach high enough $\Delta \tilde{\mu}_{\mathrm{H}^{+}}$dark to fully describe the plateau of $\mathrm{CF}_{1} \mathrm{~F}_{\mathrm{o}}$ rate. This was due to experimental constraints: in the oxidized form, it was not possible to reach higher values of $\Delta \tilde{\mu}_{\mathrm{H}^{+}}$because a sufficient dark period was needed after the $30 \mathrm{~s}$ illumination to reoxidize PSI secondary donors and PSII acceptors, as discussed before. In the reduced $\mathrm{CF}_{1} \mathrm{~F}_{\mathrm{o}}$ of the gamera mutant, illumination decreased the $\Delta \tilde{\mu}_{\mathrm{H}^{+}}$(Fig 3A). However, the observation that TCEP- ( $50 \% \gamma$-dithiol) and water-infiltrated WT leaves reached similar rates at $\Delta \tilde{\mu}_{\mathrm{H}^{+}} \sim-0.5$ charge separation/PSI (see also Fig S6, panel A) suggests that the rate of the oxidized and reduced form reached similar values in this range of $\Delta \tilde{\mu}_{\mathrm{H}^{+}}$.
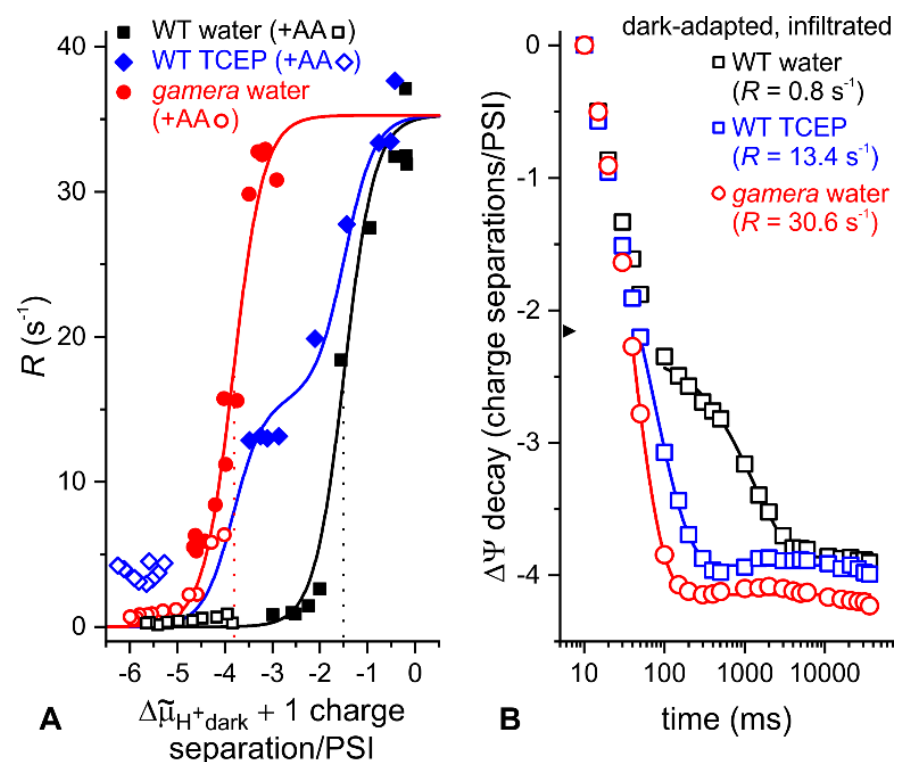

Fig 4: The regulation levels of $\Delta \tilde{\mu}_{H^{+}}$and thiol modulation on ATP synthesis are shown. (A) The $\Delta \tilde{\mu}_{H^{+}}$-dependence of the $\mathrm{CF}_{1} \mathrm{~F}_{\mathrm{o}}$ rate in the oxidized (dark) and reduced (red) forms are shown, as well as in the intermediate situation of TCEP-infiltrated WT leaves (blue). Rates were obtained according to Fig S6. Closed symbols: untreated leaves, open symbols: leaves were additionally infiltrated with $40 \mu \mathrm{M}$ antimycin-A (+AA). Fitted curves were obtained with Equation 1. The water-infiltrated WT showed a $\Delta \tilde{\mu}_{H}{ }$ activation of $\sim-2.2$ charge separations/PSI, and halfmaximal $R$ at $\sim-1.5$ charge separations/PSI (dotted black line). The $\gamma$-dithiol-containing gamera showed a halfmaximal $R$ at $\sim-3.8$ charge separations/PSI (dotted red line) and a a $\Delta \tilde{\mu}_{H^{+}}$activation of $\sim-4.7$ charge 
bioRxiv preprint doi: https://doi.org/10.1101/2020.04.28.066100; this version posted April 29, 2020. The copyright holder for this preprint (which was not certified by peer review) is the author/funder. All rights reserved. No reuse allowed without permission.

separations/PSI. (B) The decay rate $R$ (see legend, in $\mathrm{s}^{-1}$ ) of the $\mathrm{CF}_{1} \mathrm{~F}_{\mathrm{o}}$ was calculated in the range from $\Delta \tilde{\mu}_{H^{+}}$activation (arrowhead) to $\Delta \tilde{\mu}_{H^{+} \text {dark }}$ levels. The relaxation of ECS was fitted by a mono-exponential decay function. 


\section{Conclusion/ Discussion}

Taken together, the in vivo ECS protocol that was developed previously [16] allowed to estimate the $\Delta \tilde{\mu}_{\mathrm{H}^{+} \text {dark }}$ by avoiding formation of a $\Delta \mathrm{pH}$ during probing of $\Delta \Psi$. The $\Delta \tilde{\mu}_{\mathrm{H}^{+} \text {dark }}$ is defined by the $[A T P] /([A D P][P i])$ ratio and was maintained at high levels after a photophosphorylation period. It also required metabolic coupling with mitochondria which were most efficiently inhibited by uncouplers. By comparing the gamera mutant with $\mathrm{WT}$, the $\Delta \tilde{\mu}_{\mathrm{H}^{+}}$-dependent $\mathrm{CF}_{1} \mathrm{~F}_{\mathrm{o}}$ activity profile demonstrated higher efficiency of ATP synthesis in the reduced form at lower membrane energization levels. In this work, we could confirm the redox regulation of the $\mathrm{CF}_{1} \mathrm{~F}_{\mathrm{o}}$ previously described by Junesch and Gräber in vitro [36] in which a chemically reduced disulfide bond in the $\gamma$-subunit produced half-maximal rates at a lower $\Delta \tilde{\mu}_{\mathrm{H}^{+}}$of $0.7 \Delta \mathrm{pH}$ units. In our work, we obtained a very good agreement with this value since we measured that the $\Delta \tilde{\mu}_{\mathrm{H}^{+}}$required for half saturation of the $\mathrm{CF}_{1} \mathrm{~F}_{\mathrm{o}}$ was 2.3 charge separations/PSI higher in the oxidized form (WT) compared to the reduced form (gamera mutant). Accepting a value of $20 \mathrm{mV}$ per PSI charge separation $[38,48,49]$, this determined value $(\sim 45 \mathrm{mV})$ was very close to the $0.7 \mathrm{pH}$ units.

Although the $\mathrm{pH}$ shift between the two sigmoids in the in vitro work of Junesch and Gräber, and the one we obtained in vivo were in good agreement, our results do not allow to strictly compare the absolute values of the electrochemical gradient needed to reach half-saturation. This comes from the fact that the $\Delta \tilde{\mu}_{\mathrm{H}^{+}}$values in our work are expressed in relative values. For a proper in vitro and in vivo comparison, our results should be expressed in absolute values, i.e. compared to the situation where $\Delta \tilde{\mu}_{\mathrm{H}^{+} \text {dark }}$ is null.

How to reach the situation where $\Delta \tilde{\mu}_{H^{+} \text {dark }}=0$ ?

Coming back to our opaque bottle analogy, the water volume contained in an opaque bottle can be easily calculated as the difference between the empty volume of the bottle (measured as the volume of water that needs to be added before it spills out) and the maximal countenance capacity (same measurement with previously emptied bottle). Several studies $[16,46]$ have shown that the electrochemical proton gradient in the dark $\left(\Delta \tilde{\mu}_{\mathrm{H}^{+} \text {dark }}\right)$ can be collapsed by infiltrating the samples with appropriate inhibitors. This is the equivalent of the "emptied bottle" situation, and allows expressing the values of $\Delta \tilde{\mu}_{\mathrm{H}^{+} \text {dark }}$ as absolute values, like in the work of Junesch and Gräber, instead of relative values (by comparison to $\mathrm{ECS}_{10 \mathrm{~ms}}$ ). In brief, the ECS-based protocol to measure the absolute value of $\Delta \tilde{\mu}_{\mathrm{H}^{+} \text {dark }}$ is the following: 1 - energizing the membrane with a very strong light pulse and probing the ECS increase until the $\Delta \tilde{\mu}_{\mathrm{H}^{+} \text {leak }}$ is reached (i.e., measuring the "empty volume"), 2- collapsing the $\Delta \tilde{\mu}_{\mathrm{H}^{+} \text {dark }}$ with appropriate inhibitors, and 3- repeating the experiment starting from such a situation where $\Delta \tilde{\mu}_{\mathrm{H}^{+} \text {dark }}$ is null (i.e., measuring the "maximal countenance capacity"). The difference between the amplitudes of the two pulse-induced ECS increases corresponds to the absolute $\Delta \tilde{\mu}_{\mathrm{H}^{+}}$dark in the non-treated sample. To do this, one needs to find a set of inhibitors that totally collapse the $\Delta \tilde{\mu}_{\mathrm{H}^{+} \text {dark. }}$ The latter is built at the expense of the ATP present in the plastid in dark-adapted leaves, which originates from mitochondrial ATP production as has been shown previously for algae [50], diatoms [46], and higher plants [16]. In mature plant tissues, the cytosolic ATP import is relatively slow due to low abundance of nucleotide transporters in the chloroplast membrane [45]. Previous reports $[16,46]$ and the first section of this manuscript used antimycin-A (AA) treatment, which inhibits the bc1 complex in the mitochondrial respiration. This was proposed to suppress mitochondrial ATP production and therefore to collapse $\Delta \tilde{\mu}_{\mathrm{H}^{+} \text {dark }}$ [16]. However, it is known that in the absence of bc1 
activity, a mitochondrial electron flow can still occur thanks to the activity of the alternative oxidase (AOX). Despite its non-electrogenicity, the AOX allows the complex I to function and to generate an electrochemical proton gradient and mitochondrial ATP. In agreement with this, the use of AA alone does not fully collapse the electrochemical gradient in diatoms [46] and only the inhibition of both the bc1 complex (with AA) and the AOX (with salicylhydroxamic acid) fully suppressed the electrochemical gradient. To determine the best protocol to collapse the $\Delta \tilde{\mu}_{\mathrm{H}^{+} \text {dark }}$ in plants, we compared different pharmacological treatments (Fig S4). Fig S4 displays the ECS decay kinetics after a pulse in dark-

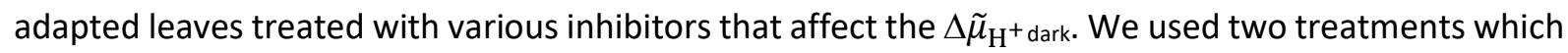

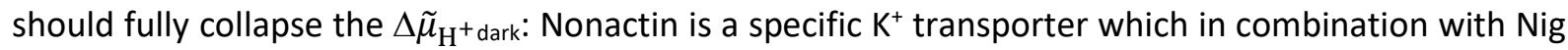
should remove both the $\Delta \Psi$ and $\Delta \mathrm{pH}$ across the thylakoid in the dark, as should FCCP, which is an uncoupler. The dark-adapted values of the $\Delta \tilde{\mu}_{\mathrm{H}^{+}}$were very similar between the two treatments as well as with the treatment with Nig alone, indicating that Nig was sufficient to suppress the mitochondrial

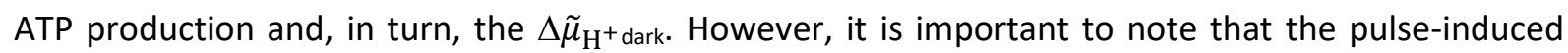
$\Delta \tilde{\mu}_{\mathrm{H}^{+}}$had a very long lifetime ( $\sim 5-60 \mathrm{~s}$ ) in Nig-infiltrated leaves. This reflected, in the absence of mitochondrial ATP supply, the interplay of Nig-dependent $\Delta \mathrm{pH} / \Delta \Psi$ exchange and the consumption of the ATP generated during the pulse. We then compared AA treatment to the Nig treatment and obtained variable results in different plants. In some cases (Fig S4B), the dark-adapted value of the $\Delta \tilde{\mu}_{\mathrm{H}^{+}}$in $\mathrm{AA}$ was very similar to the one in Nig, indicating that $\mathrm{AA}$ inhibited most of the mitochondrial ATP production whereas in other cases (Fig S4C) Nig and AA treatments gave very different results, suggesting that the activity of the AOX was still significant. To conclude, when one wants to express $\Delta \tilde{\mu}_{\mathrm{H}^{+} \text {dark }}$ in absolute values (i.e. to measure the "total volume" of the opaque bottle), the reference for $\Delta \tilde{\mu}_{\mathrm{H}^{+} \text {dark }}=0$ should be obtained with Nig, which suppresses mitochondrial ATP production, or with thylakoid uncouplers collapsing the $\Delta \tilde{\mu}_{\mathrm{H}^{+} \text {dark }}$ across the thylakoid (e.g. FCCP or the combination of Nig and nonactin). However, it is important to note that since these compounds embedded to the lipid bilayer, fast and slow ECS decay kinetics will be altered and cannot be attributed to $\mathrm{CF}_{1} \mathrm{~F}_{\mathrm{o}}$ performance alone.

With this in mind, it is possible to discuss our results in the light of the 2.7 and $3.4 \mathrm{pH}$ units for half saturation of the reduced and oxidized ATP synthesis rate in vitro [36], which would correspond here to 162 and $204 \mathrm{mV}$. With a calibration of $20 \mathrm{mV}$ per PSI charge separation, this would give 8.1 and $\sim 10.2$ charge separations/PSI. For the reduced and oxidized ATP synthesis rate in vivo, the halfsaturation was reached at a $\Delta \tilde{\mu}_{\mathrm{H}^{+} \text {dark }}$ of -3.8 and -1.5 charge separation/PSI. Here, the lowest value of $\Delta \tilde{\mu}_{\mathrm{H}^{+} \text {dark }}$ was obtained for AA-infiltrated WT leaves in the presence of TCEP, which was $\sim-7.5$ charge separations/PSI (note that $x$-axis in Fig $4 \mathrm{~A}$ is $\Delta \tilde{\mu}_{\mathrm{H}^{+}}$dark +1 ). As outlined above, the $\Delta \tilde{\mu}_{\mathrm{H}^{+} \text {dark }}$ in TCEP/AA samples was significantly higher than 0 . In fact, the remaining $\Delta \tilde{\mu}_{\mathrm{H}^{+} \text {dark }}$ in TCEP/AA should be at least 4 charge separations/PSI to explain the discrepancy $(4.2=10.2-7.5+1.5$ charge separations/PSI; 84 $\mathrm{mV}$ or $1.4 \mathrm{pH}$ units for oxidized $\mathrm{CF}_{1} \mathrm{~F}_{\mathrm{o}}$ ). Such a value being high according to the results of Fig $\mathrm{S} 4$, we acknowledge here a slight discrepancy between our results and the ones obtained in vitro [36].

\section{What does the lifetime of ATP depend on?}

In this work, we could measure the lifetime of ATP after an illumination and showed in section 3 that the ATP accumulated after an illumination saturates after $7 \mathrm{~s}$ of illumination, and that longer illumination durations lead to a lag before the ATP pool relaxes. We favor the hypothesis that the increased ATP lifetime (closed squares in Fig $3 \mathrm{~A}$ ), as a function of the pre-illumination period, was associated with the accumulation of high energy bonds in different plastid metabolites. This hypothesis 
is supported in a nucleotide transporter study which revealed that the ATP exchange rate between chloroplast and cytosol was rather slow, and fluorescent ATP probes suggested that excess of free ATP during illumination diminished rapidly in the dark [45]. Thus, lowering the $\Delta \tilde{\mu}_{\mathrm{H}^{+} \text {dark }}$ in Fig $3 \mathrm{~A}$ would be delayed after prolonged illumination due to relatively slow metabolite conversions that consume ATP in the dark. For instance, high levels of ribulose-1,5-bisphosphate and 1,3-bisphosphoglycerate in the Calvin-Benson cycle could be part of a feedback inhibition that prevents their own ATP-consuming synthesis. Furthermore, the deactivation of the Calvin-Benson cycle in the dark [16] was in the same

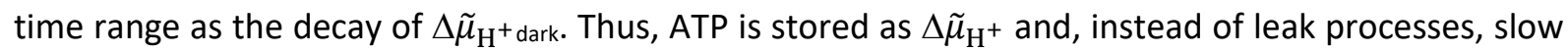
metabolic consumption occurs within the chloroplast. When infiltrating leaves with TCEP (midpoint redox potential of $-290 \mathrm{mV}$ [51]), various thiol enzymes are expected to be partially reduced due to the chemical which could lead to their activation. Usually catalyzed by reduced thioredoxin, TCEP could activate Calvin-Benson cycle enzymes such as fructose-1,6-bisphosphatase or sedoheptulose-1,7bisphosphatase. This, in turn, could explain a slightly earlier onset of the consumption of ATP after 30$s$ illumination in TCEP-infiltrated samples (cf. open squares in Fig $3 A$ ), which we measured on the $\Delta \tilde{\mu}_{\mathrm{H}^{+} \text {dark }}$ levels.

Is the dark-adapted $\mathrm{CF}_{1} F_{\text {o }}$ fully oxidized?

In the fit of the water-infiltrated experimental data (black curve, Fig 4A), we considered that the darkadapted $\mathrm{CF}_{1} \mathrm{~F}_{\mathrm{o}}$ was fully oxidized. Nevertheless, about $5 \%$ of $\mathrm{CF}_{1} \mathrm{~F}_{\mathrm{o}}$ were reduced in a previous study of dark-adapted spinach chloroplasts [2], suggesting that $\gamma$-dithiols in water-infiltrated samples were likely not fully oxidized. Also, the rate we measured in the low $\Delta \tilde{\mu}_{\mathrm{H}^{+}}$range (Fig $4 \mathrm{~B}$ ) was not null although it was previously proposed that the $\mathrm{CF}_{1} \mathrm{~F}_{\mathrm{o}}$ was fully inactive below the $\Delta \tilde{\mu}_{\mathrm{H}^{+} \text {activation }}[10,11]$. If so, the rate constants measured in Fig $4 \mathrm{~B}\left(0.8 \mathrm{~s}^{-1}\right.$ in control vs. $30.6 \mathrm{~s}^{-1}$ in gamera) would mean that in our case, $2-3 \%$ of the $\mathrm{CF}_{1} \mathrm{~F}_{\mathrm{o}}$ is in the reduced form in the dark-adapted state. At $\Delta \tilde{\mu}_{\mathrm{H}^{+}}$dark levels below the $\Delta \tilde{\mu}_{\mathrm{H}^{+}}$activation, the contribution of ion channel activity to the ECS decay was likely more significant in WT leaves.

\section{Uncoupler effect in TCEP-infiltrated leaves?}

In Fig 4A, the fit of the TCEP-infiltrated experimental data by the sum of two sigmoids failed for the AA-treated samples: significantly elevated rates were observed when the $\Delta \tilde{\mu}_{\mathrm{H}^{+}}$dark was low in the presence of TCEP + AA (open blue diamonds). We cannot rule out that TCEP infiltration had a weak uncoupling effect or modified anion channel activity, thus producing slightly faster $\Delta \Psi$ decay rates. This effect would slightly overestimate the TCEP-dependent rates in Fig 4A. In line with this, the slightly earlier decrease of $\Delta \tilde{\mu}_{\mathrm{H}^{+} \text {dark }}$ in TCEP-infiltrated samples upon illumination might be due to a slow leak process (Fig 3A). The leak might be due to activation of anion channels or deactivation of $\mathrm{H}^{+} / \mathrm{cation}$ antiporters. It has also been reported that DTT treatment of chloroform-extracted $\mathrm{CF}_{1}$ resulted in the reversible dissociation of the $\varepsilon$-subunit $[52,53]$ which, if this is the case for $\mathrm{CF}_{1} \mathrm{~F}_{0}$ in vivo, would produce uncoupling [54]. It remains to be tested whether the elevated ECS decay rates in the presence of TCEP, most pronounced at low $\Delta \tilde{\mu}_{\mathrm{H}^{+} \text {dark }}$ levels (open blue diamonds in Fig $4 \mathrm{~A}$ ), were due to ion channels other than $\mathrm{CF}_{1} \mathrm{~F}_{\mathrm{o}}$.

\section{Combining function, structure and physiology}

In the light of the recently obtained structure of the $\mathrm{CF}_{1} \mathrm{~F}_{\mathrm{o}}$, we would like to try linking our results to structural considerations. The L-shaped double hairpin (Fig 5A, light green in magnified area) in the disulfide-containing spinach $\mathrm{CF}_{1} \mathrm{~F}_{0}$ structure interacts with the $\beta$-subunit DELSEED motif and has 
therefore been suggested to prevent ATP hydrolysis by forming a chock during rotation [3]. Steric interference of rotation in the opposite direction (ATP synthesis) can thus be proposed for this structural element as well. The $\gamma$-hairpin and the $\beta$-DELSEED loop harbor various residues that are not surface-exposed (e.g. $\gamma$ K222 and $\beta 399$ in Fig $5 A$ ) but have been chemically modified in illuminated thylakoids only [27, 28, reviewed in 29]. Thus, the structural impact of the $\Delta \tilde{\mu}_{\mathrm{H}^{+}}$on $\mathrm{CF}_{1} \mathrm{~F}_{\mathrm{o}}$ is occurring (among other sites) in the rotational chock element. Solvent exposure of the latter should reduce the bulk of rotating $\gamma$-residues in $\Delta \tilde{\mu}_{\mathrm{H}^{+}}$-activated $\mathrm{CF}_{1} \mathrm{~F}_{\mathrm{o}}$ which modify the sum of van der Waals contacts with the $\beta$-DELSEED loop. This $\beta$-subunit loop moves perpendicular to the $\gamma$-subunit axis and the stroke movements (exerted by $\gamma$ and $\beta$ during ATP synthesis and hydrolysis, respectively) eventually change the binding site conformation and thus the nucleotide affinity [55]. The sequential binding site cooperativity is most efficient in $\Delta \tilde{\mu}_{\mathrm{H}^{+}}$-activated $\mathrm{CF}_{1} \mathrm{~F}_{\mathrm{o}}$, yielding maximal rates of the enzyme in both redox states (Fig $5 \mathrm{~B}$ above dashed line). When the $\Delta \tilde{\mu}_{\mathrm{H}^{+}}$approaches lower levels, the choc structure becomes buried again and rotational catalysis occurs at lower efficiency (Fig 5B WT below dashed line). The L-shaped double hairpin position is stabilized by the $\gamma$-disulfide (Fig 5A, yellow in magnified area) which might explain why the reduced enzyme remains in a highly active state at lower $\Delta \tilde{\mu}_{\mathrm{H}^{+}}$(Fig $5 \mathrm{~B}$ gamera below dashed line). From a physiological viewpoint, enabling higher $\mathrm{CF}_{1} \mathrm{~F}_{\mathrm{o}}$ rates for a given $\Delta \tilde{\mu}_{\mathrm{H}^{+}}$level upon $\gamma$-disulfide cleavage has the advantage to maintain a high $\mathrm{H}^{+}$conductivity via $\mathrm{CF}_{1} \mathrm{~F}_{\mathrm{o}}$ even in sub-saturating light conditions. Thus, ATP production is maximized and electron transfer limitation (triggered by an acidic lumen for instance) can be avoided. Probably therefore, disulfide cleavage in vivo is very efficiently realized in $\operatorname{dim}$ light $[19,20]$ and at higher light intensities $[21-23,28$, 56].

A

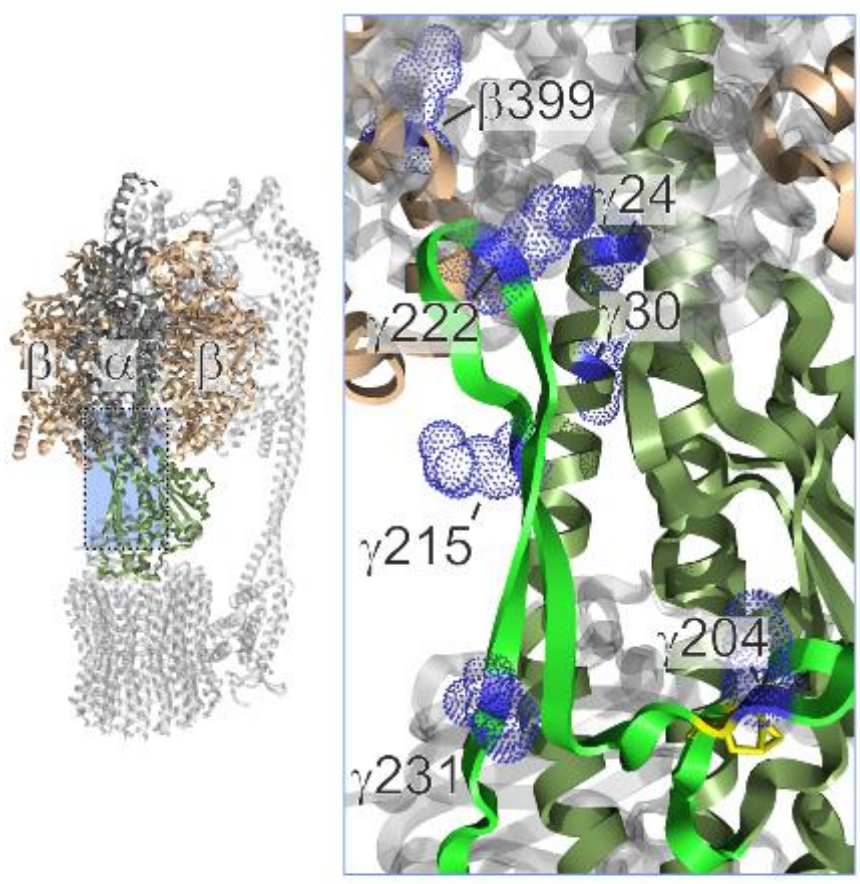

Fig 5: A selection of $\Delta \tilde{\mu}_{H^{+}}$-dependent structural rearrangements in spinach $\mathrm{CF}_{1} \mathrm{~F}_{\mathrm{o}}$ and a tentative model for different ATP synthesis rates as a function of $\Delta \tilde{\mu}_{H^{+}}$are shown. (A) The structure of spinach $\mathrm{CF}_{1} \mathrm{~F}_{\mathrm{o}}$ (PDB ID 6fkh) magnifies the redox loop (light green, disulfide in yellow sticks) on the right. The frontal $\alpha$-subunit is magnified half-transparent for the sake of visibility of the $\gamma$-subunit (dark green). The ATPase choc structure is formed by 
bioRxiv preprint doi: https://doi.org/10.1101/2020.04.28.066100; this version posted April 29, 2020. The copyright holder for this preprint (which was not certified by peer review) is the author/funder. All rights reserved. No reuse allowed without permission.

the $\gamma$-hairpin loop around position 222 which is stabilized by the disulfide [3]. A variety of structural rearrangements are expected depending on the $\Delta \tilde{\mu}_{H^{+}}$, including the $\gamma$-hairpin. Critical residues (blue spheres) become available for chemical labeling or trypsin digestion exclusively in illuminated thylakoids [reviewed in 29]. (B) The exposed position of the choc structure in $\Delta \tilde{\mu}_{H^{+}}$-activated $\mathrm{CF}_{1} \mathrm{~F}_{\mathrm{o}}$ allows efficient rotational catalysis in both $\gamma$-redox states (top). Below the WT activation threshold (dashed line), the disulfide-stabilized choc structure prevents efficient ATP synthesis. The gamera sustains an active conformation at this $\Delta \tilde{\mu}_{H^{+}}$since the choc structure is less efficiently fixed inside the $\alpha_{3} \beta_{3}$ cavity, owing to the absence of the $\gamma$-disulfide. 
bioRxiv preprint doi: https://doi.org/10.1101/2020.04.28.066100; this version posted April 29, 2020. The copyright holder for this preprint (which was not certified by peer review) is the author/funder. All rights reserved. No reuse allowed without permission.

Acknowledgements: We would like to thank Dr. Jörg Meurer (Ludwig Maximilian University of Munich) for sharing the dpa1c and gamera seeds with us. B.B. and F.B. acknowledge funding from the ERC Starting Grant PhotoPHYTOMICS (ERC-2016-STG grant \# 715579). F.B. also acknowledge the CNRS and the "Initiative d' Excellence" Program from the French state grant "DYNAMO", ANR-11-LABX-0011-01. 
bioRxiv preprint doi: https://doi.org/10.1101/2020.04.28.066100; this version posted April 29, 2020. The copyright holder for this preprint (which was not certified by peer review) is the author/funder. All rights reserved. No reuse allowed without permission.

Reference list

[1] W. Junge, N. Nelson, ATP synthase, Annu. Rev. Biochem., 84 (2015) 631-657.

[2] T. Hisabori, E.I. Sunamura, Y. Kim, H. Konno, The chloroplast ATP synthase features the characteristic redox regulation machinery, Antioxidants \& Redox Signaling, 19 (2013) 1846-U1216.

[3] A. Hahn, J. Vonck, D.J. Mills, T. Meier, W. Kuhlbrandt, Structure, mechanism, and regulation of the chloroplast ATP synthase, Science, 360 (2018).

[4] R.E. McCarty, J.S. Fuhrman, Y. Tsuchiya, Effects of adenine nucleotides on hydrogen-ion transport in chloroplasts, Proc Natl Acad Sci U S A, 68 (1971) 2522-2526.

[5] G. Groth, W. Junge, Proton slip of the chloroplast ATPase: its nucleotide dependence, energetic threshold, and relation to an alternating site mechanism of catalysis, Biochemistry, 32 (1993) 81038111.

[6] P. Turina, J. Petersen, P. Graber, Thermodynamics of proton transport coupled ATP synthesis, Biochim. Biophys. Acta, 1857 (2016) 653-664.

[7] A. Kanazawa, D.M. Kramer, In vivo modulation of nonphotochemical exciton quenching (NPQ) by regulation of the chloroplast ATP synthase, Proc. Natl. Acad. Sci. USA, 99 (2002) 12789-12794.

[8] K. Takizawa, A. Kanazawa, D.M. Kramer, Depletion of stromal Pi induces high 'energy-dependent' antenna exciton quenching $\left(\mathrm{q}_{\mathrm{E}}\right)$ by decreasing proton conductivity at CFo-CF1 ATP synthase, Plant Cell Environ, 31 (2008) 235-243.

[9] F. Buchert, B. Bailleul, T. Hisabori, A $\gamma$-subunit point mutation in Chlamydomonas reinhardtii chloroplast $F_{1} F_{0}$-ATP synthase confers tolerance to reactive oxygen species, Biochim. Biophys. Acta, 1858 (2017) 966-974.

[10] W. Junge, B. Rumberg, H. Schroder, The necessity of an electric potential difference and its use for photophosphorylation in short flash groups, Eur. J. Biochem., 14 (1970) 575-581.

[11] W. Junge, The critical electric potential difference for photophosphorylation. Its relation to the chemiosmotic hypothesis and to the triggering requirements of the ATPase system, Eur. J. Biochem., 14 (1970) 582-592.

[12] J.H. Kaplan, E. Uribe, A.T. Jagendorf, ATP hydrolysis caused by acid-base transition of spinach chloroplasts, Arch Biochem Biophys, 120 (1967) 365-370.

[13] P. Gräber, H.T. Witt, Relations between the electrical potential, pH gradient, proton flux and phosphorylation in the photosynthetic membrane, Biochim. Biophys. Acta, 423 (1976) 141-163.

[14] D.A. Harris, A.R. Crofts, The initial stages of photophosphorylation. Studies using excitation by saturating, short flashes of light, Biochim. Biophys. Acta, 502 (1978) 87-102.

[15] D.M. Kramer, A.R. Crofts, Activation of the chloroplast ATPase measured by the electrochromic change in leaves of intact plants, Biochimica et Biophysica Acta, 976 (1989) 28-41.

[16] P. Joliot, A. Joliot, Quantification of the electrochemical proton gradient and activation of ATP synthase in leaves, Biochim. Biophys. Acta, 1777 (2008) 676-683.

[17] K. Yoshida, A. Hara, K. Sugiura, Y. Fukaya, T. Hisabori, Thioredoxin-like2/2-Cys peroxiredoxin redox cascade supports oxidative thiol modulation in chloroplasts, Proc. Natl. Acad. Sci. USA, 115 (2018) E8296-E8304.

[18] J.D. Mills, P. Mitchell, P. Schurmann, Modulation of coupling factor ATPase activity in intact chloroplasts - The role of the thioredoxin system, FEBS Lett., 112 (1980) 173-177.

[19] B. Naranjo, C. Mignee, A. Krieger-Liszkay, D. Hornero-Mendez, L. Gallardo-Guerrero, F.J. Cejudo, M. Lindahl, The chloroplast NADPH thioredoxin reductase C, NTRC, controls non-photochemical quenching of light energy and photosynthetic electron transport in Arabidopsis, Plant Cell Environ, 39 (2016) 804-822.

[20] L.R. Carrillo, J.E. Froehlich, J.A. Cruz, L.J. Savage, D.M. Kramer, Multi-level regulation of the chloroplast ATP synthase: the chloroplast NADPH thioredoxin reductase C (NTRC) is required for redox modulation specifically under low irradiance, Plant J., 87 (2016) 654-663.

[21] S.R. Ketcham, J.W. Davenport, K. Warncke, R.E. McCarty, Role of the $\gamma$ subunit of chloroplast coupling factor- 1 in the light-dependent activation of photophosphorylation and ATPase activity by dithiothreitol, J. Biol. Chem., 259 (1984) 7286-7293. 
[22] H. Konno, T. Nakane, M. Yoshida, H. Ueoka-Nakanishi, S. Hara, T. Hisabori, Thiol modulation of the chloroplast ATP synthase is dependent on the energization of thylakoid membranes, Plant Cell Physiol., 53 (2012) 626-634.

[23] K. Yoshida, Y. Matsuoka, S. Hara, H. Konno, T. Hisabori, Distinct redox behaviors of chloroplast thiol enzymes and their relationships with photosynthetic electron transport in Arabidopsis thaliana, Plant Cell Physiol., 55 (2014) 1415-1425.

[24] L. Nikkanen, J. Toivola, A. Trotta, M. Guinea Diaz, M. Tikkanen, E.-M. Aro, E. Rintamaki, Regulation of chloroplast NADH dehydrogenase-like complex by NADPH-dependent thioredoxin system, bioRxiv, (2018).

[25] J.V. Moroney, C.S. Andreo, R.H. Vallejos, R.E. McCarty, Uncoupling and energy transfer inhibition of photophosphorylation by sulfhydryl reagents, J. Biol. Chem., 255 (1980) 6670-6674.

[26] M. Komatsu-Takaki, Energy-dependent conformational changes in the $\varepsilon$ subunit of the chloroplast ATP synthase $\left(\mathrm{CF}_{0} \mathrm{CF}_{1}\right)$, J. Biol. Chem., 264 (1989) 17750-17753.

[27] M. Komatsu-Takaki, Effects of Energization and Substrates on the Reactivities of Lysine Residues of the Chloroplast ATP Synthase $\beta$ Subunit, Eur. J. Biochem., 228 (1995) 265-270.

[28] M. Komatsu-Takaki, Energizing effects of illumination on the reactivities of lysine residues of the $\gamma$ subunit of chloroplast ATP synthase, Eur. J. Biochem., 236 (1996) 470-475.

[29] M.L. Richter, R. Hein, B. Huchzermeyer, Important subunit interactions in the chloroplast ATP synthase, Biochim. Biophys. Acta, 1458 (2000) 326-342.

[30] J.Z. Pu, M. Karplus, How subunit coupling produces the $\gamma$ subunit rotary motion in $\mathrm{F}_{1}$-ATPase, Proc. Natl. Acad. Sci. USA, 105 (2008) 1192-1197.

[31] D.R. Ort, K. Oxborough, In situ regulation of chloroplast coupling factor activity, Annu. Rev. Plant Physiol. Plant Mol. Biol., 43 (1992) 269-291.

[32] G. Wu, D.R. Ort, Mutation in the cysteine bridge domain of the $\gamma$ subunit affects light regulation of the ATP synthase but not photosynthesis or growth in Arabidopsis, Photosynthesis Res., 97 (2008) 185-193.

[33] K. Kohzuma, C. Dal Bosco, A. Kanazawa, A. Dhingra, W. Nitschke, J. Meurer, D.M. Kramer, Thioredoxin-insensitive plastid ATP synthase that performs moonlighting functions, Proc. Natl. Acad. Sci. USA, 109 (2012) 3293-3298.

[34] K. Kohzuma, J.E. Froehlich, G.A. Davis, J.A. Temple, D. Minhas, A. Dhingra, J.A. Cruz, D.M. Kramer, The Role of Light-Dark Regulation of the Chloroplast ATP Synthase, Frontiers in Plant Science, 8 (2017) 1248.

[35] F. Buchert, H. Konno, T. Hisabori, Redox regulation of $\mathrm{CF}_{1}-\mathrm{ATPase}$ involves interplay between the $\gamma$-subunit neck region and the turn region of the $\beta$ DELSEED-loop, Biochim. Biophys. Acta, 1847 (2015) 441-450.

[36] U. Junesch, P. Graber, Influence of the redox state and the activation of the chloroplast ATP synthase on proton-transport-coupled ATP synthesis hydrolysis, Biochim. Biophys. Acta, 893 (1987) 275-288.

[37] W. Junge, H.T. Witt, On the ion transport system of photosynthesis--investigations on a molecular level, Z Naturforsch B, 23 (1968) 244-254.

[38] H.T. Witt, Energy conversion in the functional membrane of photosynthesis. Analysis by light pulse and electric pulse methods. The central role of the electric field, Biochim. Biophys. Acta, 505 (1979) 355-427.

[39] B. Bailleul, P. Cardol, C. Breyton, G. Finazzi, Electrochromism: a useful probe to study algal photosynthesis, Photosynthesis Res., 106 (2010) 179-189.

[40] G. Wu, G. Ortiz-Flores, A. Ortiz-Lopez, D.R. Ort, A point mutation in atpC1 raises the redox potential of the Arabidopsis chloroplast ATP synthase $\gamma$ subunit regulatory disulfide above the range of thioredoxin modulation, J. Biol. Chem., 282 (2007) 36782-36789.

[41] W. Junge, W. Auslander, A.J. McGeer, T. Runge, The buffering capacity of the internal phase of thylakoids and the magnitude of the $\mathrm{pH}$ changes inside under flashing light, Biochim. Biophys. Acta, 546 (1979) 121-141. 
[42] C. Lemaire, F.A. Wollman, P. Bennoun, Restoration of phototrophic growth in a mutant of Chlamydomonas reinhardtii in which the chloroplast atpB gene of the ATP synthase has a deletion: an example of mitochondria-dependent photosynthesis, Proc. Natl. Acad. Sci. USA, 85 (1988) 1344-1348. [43] J. Reiser, N. Linka, L. Lemke, W. Jeblick, H.E. Neuhaus, Molecular physiological analysis of the two plastidic ATP/ADP transporters from Arabidopsis, Plant Physiol., 136 (2004) 3524-3536.

[44] A.P. Weber, N. Linka, Connecting the plastid: transporters of the plastid envelope and their role in linking plastidial with cytosolic metabolism, Annu. Rev. Plant Biol., 62 (2011) 53-77.

[45] C.P. Voon, X. Guan, Y. Sun, A. Sahu, M.N. Chan, P. Gardestrom, S. Wagner, P. Fuchs, T. Nietzel, W.K. Versaw, M. Schwarzlander, B.L. Lim, ATP compartmentation in plastids and cytosol of Arabidopsis thaliana revealed by fluorescent protein sensing, Proc. Natl. Acad. Sci. USA, 115 (2018) E10778-E10787. [46] B. Bailleul, N. Berne, O. Murik, D. Petroutsos, J. Prihoda, A. Tanaka, V. Villanova, R. Bligny, S. Flori, D. Falconet, A. Krieger-Liszkay, S. Santabarbara, F. Rappaport, P. Joliot, L. Tirichine, P.G. Falkowski, P. Cardol, C. Bowler, G. Finazzi, Energetic coupling between plastids and mitochondria drives $\mathrm{CO}_{2}$ assimilation in diatoms, Nature, 524 (2015) 366-369.

[47] D.J. Cline, S.E. Redding, S.G. Brohawn, J.N. Psathas, J.P. Schneider, C. Thorpe, New water-soluble phosphines as reductants of peptide and protein disulfide bonds: reactivity and membrane permeability, Biochemistry, 43 (2004) 15195-15203.

[48] W. Schliephake, W. Junge, H.T. Witt, Correlation between field formation, proton translocation, and the light reactions in photosynthesis, Z Naturforsch B, 23 (1968) 1571-1578.

[49] A.A. Bulychev, W.J. Vredenberg, Effect of ionophores A23187 and nigericin on the light-induced redistribution of $\mathrm{Mg}^{2+}, \mathrm{K}^{+}$and $\mathrm{H}^{+}$across the thylakoid membrane, Biochim. Biophys. Acta, 449 (1976) 48-58.

[50] P. Bennoun, Chlororespiration revisited: Mitochondrial-plastid interactions in Chlamydomonas, Biochim. Biophys. Acta, 1186 (1994) 59-66.

[51] P.K. Pullela, T. Chiku, M.J. Carvan, 3rd, D.S. Sem, Fluorescence-based detection of thiols in vitro and in vivo using dithiol probes, Anal. Biochem., 352 (2006) 265-273.

[52] R.J. Duhe, B.R. Selman, The dithiothreitol-stimulated dissociation of the chloroplast coupling factor-1 $\varepsilon$ subunit is reversible, Biochim. Biophys. Acta, 1017 (1990) 70-78.

[53] R.J. Duhe, B.R. Selman, Studies on the heterogeneity of the soluble chloroplast coupling factor 1 : The formation of $\varepsilon$-deficient isozymes, Biochim. Biophys. Acta, 974 (1989) 294-302.

[54] M.L. Richter, W.J. Patrie, R.E. McCarty, Preparation of the $\varepsilon$ subunit and $\varepsilon$ subunit-deficient chloroplast coupling factor-1 in reconstitutively active forms, J. Biol. Chem., 259 (1984) 7371-7373.

[55] R. Watanabe, K. Koyasu, H. You, M. Tanigawara, H. Noji, Torque transmission mechanism via DELSEED loop of $F_{1}$-ATPase, Biophys. J., 108 (2015) 1144-1152.

[56] O. Fritsche, W. Junge, Chloroplast ATP synthase: The clutch between proton flow and ATP synthesis is at the interface of subunit gamma and CF1, Bba-Bioenergetics, 1274 (1996) 94-100. 

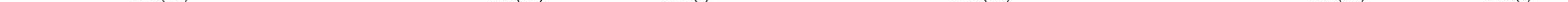
time (ms) time (s) time $(\mathrm{ms})$ 


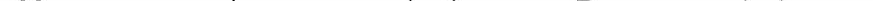




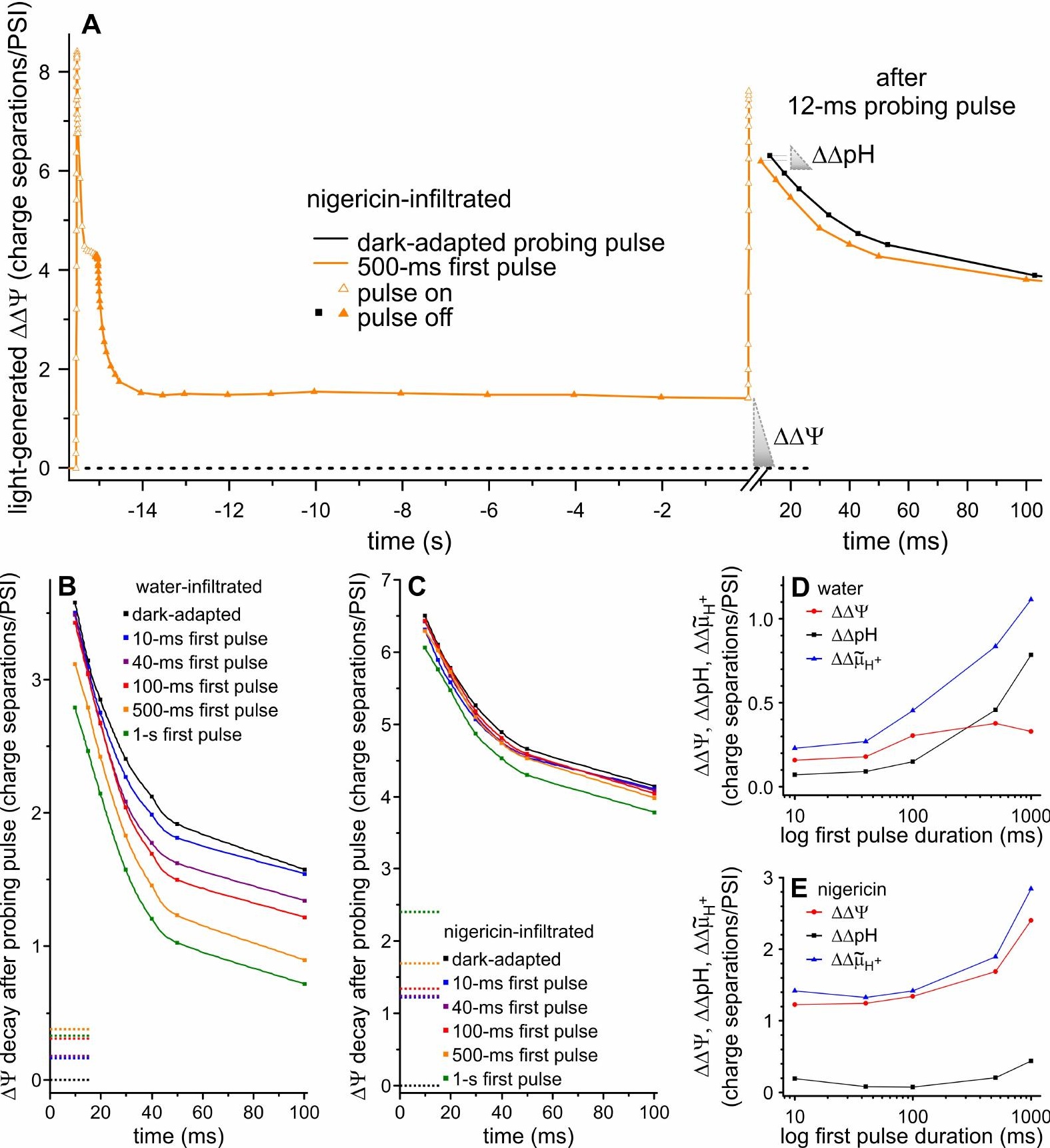

